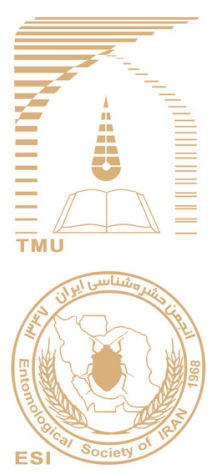

\section{A survey of True flies (Insecta: Diptera) by DNA Barcoding of Malaise Trap Collection in Bangladesh}

\author{
Santosh Mazumdar ${ }^{*}$, Paul D.N. Hebert²®, Badrul Amin Bhuiya ${ }^{3}$ \\ 1 Department of Zoology, University of Chittagong, Bangladesh. mazumdarsantosh@gmail.com \\ 2 Centre for Biodiversity Genomics, University of Guelph, 50 Stone Road East, Guelph, Ontario, Canada. \\ pheber@@uoguelph.ca \\ 3 Biodiversity Research for Environment \& Ecosystem Protection (BREEP), Chattogram-4325, Bangladesh. \\ badrulbhuiya@yahoo.com
}

A BSTRACT. True flies (Insecta: Diptera) are widely distributed and the key components in most ecosystems. The objective of this study was to identify the true flies' diversity through DNA barcoding technique (658 bp sequence from the 5'-end of cytochromeoxidase I) in Bangladesh. Specimens were collected

Received:

13 August, 2020

Accepted:

11 October, 2020

Published:

15 October, 2020

Subject Editor:

Ebrahim Gilasian by a Malaise trap at Chittagong University Campus between April 2014 and March 2015. In this study, we examined 36476 sequences of 38267 true flies, and resulted 105 species, 109 genera, 54 subfamilies and 59 families. Among them 79 species, 69 genera, 12 subfamilies and 23 families are new country records. All the specimen records with the Barcode Index Numbers (BINs) (the species proxies), are available on the Barcode of Life Data System (BOLD).

Key words: Diptera, Malaise trap, DNA barcode, Bangladesh

Citation: Mazumdar, S., Hebert, P.D.N. \& Bhuiya, B.A. (2021) A survey of True flies (Insecta: Diptera) by DNA

Barcoding of Malaise Trap Collection in Bangladesh. Journal of Insect Biodiversity and Systematics, 7 (1), 15-42.

\title{
Introduction
}

Diptera (True flies) is one of the third largest insect order containing approximately 125,000 described species (ADCF, 2020). They are effective contributors in maintaining ecological balance and in considerable economic status. For instance, they play significant positive role as scavengers, parasitoids and predators of other insects, pollinators, food for predators, bioindicators of water quality, and tools for scientific research as well as effects on agriculture, animal and human health, and forestry (Courtney et al., 2017; Dhamorikar, 2017).

True flies' taxonomic works in Bangladesh has mainly focused morphological characters, by considering their medical and agriculture importance. For example, Huda (1981) worked on genus Anopheles from Chittagong University Campus (CUC) and its adjacent areas. Also, Huda (2000) reported species diversity of genus Aedes through the country. Gapud (1992) compiled true flies associated with agricultural fields. Alam et al. (2012) reported true flies of Bandarban District. Alam et al. (2009) worked on vector species of kala-azar. Pollinating true flies and their foraging behavior were assessed by Mazumdar et al. (2010, 2011). Bashar et al. (2016) worked on true flies of semi-urban areas of Dhaka city. Irish et al. (2016) reviewed articles on mosquitoes occurred in Bangladesh.

Corresponding author: Santosh Mazumdar, E-mail: mazumdarsantosh@gmail.com

Copyright (C) 2021, Mazumdar et al. This is an open access article distributed under the terms of the Creative Commons Attribution License (CC BY 4.0), which permits unrestricted use, distribution, and reproduction in any medium, provided the original author and source are credited. 
True flies species diversity has far not been well surveyed in Bangladesh applying DNA barcoding technique. For instance, Leblanc et al. (2013), Amin et al. (2014) and Alam et al. (2015) identified some agricultural pests, Rain et al. (2019), Siddiki et al. (2019) and Mazumdar (2020) studied medically and veterinary important true flies through DNA barcoding technique.

Species identification based on DNA sequences was introduced by Hebert et al. (2003) and this modern technique has revealed an effective and credible modern tool in taxonomy, evolutionary biology and biodiversity research (Hebert et al., 2016; Lin et al., 2018). DNA barcoding of arthropods collected by Malaise traps has captured the headlines for assessing terrestrial insect diversity (deWaard et al., 2019; Mazumdar et al., 2019). A research work for insect inventories result that about $75 \%$ dipterans of total specimens collections through Malaise trap in Sweden (Karlsson et al., 2020).

Therefore, the present work was carried out to disclose the partial mitochondrial COI gene sequences of the true flies' diversity available in Bangladesh.

\section{Material and methods}

\section{Specimen Collection, Processing, Identification and Specimen Deposition}

Specimens were collected by a Townes-style Malaise trap (BioQuip Inc. USA) installed in perceived flight paths at Chittagong University Campus (Lat. 22.46359 ${ }^{\circ} \mathrm{N}$; Long. $91.7808^{\circ}$ E) in Bangladesh by following the Standard Operating Protocol for the Global Malaise Trap Program (www.dnabarcoding.ca.). Between March 2014 and February 2015, the samples were harvested weekly in a $500 \mathrm{~mL}$ plastic Nalgene bottle that was filled with $375 \mathrm{~mL}$ of $95 \%$ ethanol and placed in $500 \mathrm{~mL}$ of fresh ethanol before storage at $-20^{\circ} \mathrm{C}$ until analysis. Collected insects were analyzed by following standard barcoding protocols (http://ccdb.ca/resources.php), at the Canadian Centre for DNA Barcoding (CCDB) within the Centre for Biodiversity Genomics (CBG), University of Guelph, Canada. Collection data, voucher information and taxonomy for each specimen are available in the Barcode of Life Data Systems (http://v3.boldsystems.org/index.php/TaxBrowser_Taxonpage?taxid=127). All the specimens analyzed in this study have been curated at the CBG, University of Guelph, Guelph, Ontario, Canada.

\section{Molecular Analysis and Data Analysis}

A small portion of each specimen (usually 1-3 legs) was removed and used for DNA extraction and from the whole body of smaller taxa, and vouchers were recovered after DNA extraction for imaging and curation. Tissue lysis, DNA extraction, PCR amplification, cycle sequencing and sequence analysis were performed at the CCDB following its standard protocols.

PCR amplification of COI-5' was performed with primers C_LepFolF and C_LepFolR (http://www.ccdb.ca/docs/CCDB_PrimerSets). By following PCR conditions, $94^{\circ} \mathrm{C}(1$ min), 5 cycles at $94^{\circ} \mathrm{C}(40 \mathrm{~s}), 45^{\circ} \mathrm{C}(40 \mathrm{~s}), 72^{\circ} \mathrm{C}(1 \mathrm{~min}) ; 35$ cycles at $94^{\circ} \mathrm{C}(40 \mathrm{~s}), 51^{\circ} \mathrm{C}(40 \mathrm{~s})$, $72^{\circ} \mathrm{C}(1 \mathrm{~min})$ and a final extension at $72^{\circ} \mathrm{C}(5 \mathrm{~min})$ and amplicons were sequenced using BigDye v3.1 (Applied Biosystems) on an ABI 3730XL. Sequences were assembled, aligned, and edited using CodonCode Aligner (CodonCode Corporation, USA) and submitted to Barcode of Life Data Systems (BOLD) (www.boldsystems.org). With a few exceptions, by considering sequence matches to records on BOLD, the specimens with barcodes were assigned to 59 families. 


\section{Results}

In current study, 36476 sequences were examined of 38267 true flies. Total 105 true fly species, 109 genera, 54 subfamilies and 59 families of Malaise trap collections were recorded through DNA barcoding technique. Among them 79 species, 69 genera, 12 subfamilies and 23 families were new country records for the fauna of Bangladesh (Table 1). Muscidae was the most dominant diversified family (subfamily: 3, genera: 11, species: 19) of the total count. Chironomidae showed next highest dominance (subfamily: 2, genera: 10, species: 7), followed by Agromyzidae (subfamily: 2, genera: 7, species: 7), Culicidae (subfamily: 2, genera: 5, species: 12) and Ceratopogonidae (subfamily: 2, genera: 2, species: 10). No species were confirmed in newly recorded 24 families such as Anthomyzidae, Asteiidae, Bibionidae, Carnidae, Chyromyidae, Clusiidae, Cryptochetidae, Empididae, Hippoboscidae, Keroplatidae, Lauxaniidae, Mycetophilidae, Mythicomyiidae, Neriidae, Odiniidae, Periscelididae, Platypezidae, Platystomatidae, Psilidae, Rhinophoridae, Rhinophoridae, Scatopsidae, Simuliidae, Therevidae and Xylomyidae.

Table 1. Dipterans revealed by DNA barcoding of Malaise trap samples collected in Bangladesh.

\begin{tabular}{|c|c|c|c|}
\hline Family & Subfamily & Scientific name & References \\
\hline \multirow[t]{8}{*}{ Agromyzidae } & Agromyzinae & $\begin{array}{l}\text { Melanagromyza cleomae Spencer, } \\
1961 \text { (Fig. 1) }\end{array}$ & Current study \\
\hline & & $\begin{array}{l}\text { Melanagromyza obtusa (Malloch, } \\
\text { 1914) (Fig. 2) }\end{array}$ & $\begin{array}{l}\text { Mazumdar \& } \\
\text { Bhuiya, } 2014\end{array}$ \\
\hline & & $\begin{array}{l}\text { Ophiomyia phaseoli (Tryon, 1895) } \\
\text { (Fig. 3) }\end{array}$ & $\begin{array}{l}\text { Mazumdar \& } \\
\text { Bhuiya, } 2014\end{array}$ \\
\hline & Phytomyzinae & $\begin{array}{l}\text { Calycomyza lantanae (Frick, 1956) } \\
\text { (Fig. 4) }\end{array}$ & Current study \\
\hline & & $\begin{array}{l}\text { Chromatomyia horticola (Goureau, } \\
\text { 1851) (Fig. 5) }\end{array}$ & Current study \\
\hline & & $\begin{array}{l}\text { Liriomyza sativae Blanchard, } 1938 \\
\text { (Fig. 6) }\end{array}$ & $\begin{array}{l}\text { Mazumdar \& } \\
\text { Bhuiya, } 2014\end{array}$ \\
\hline & & Phytoliriomyza sp. & Current study \\
\hline & & $\begin{array}{l}\text { Phytomyza thalictrella Spencer, } 1981 \\
\text { (Fig. 7) }\end{array}$ & Current study \\
\hline Anthomyiidae & - & - & Miah et al., 1997 \\
\hline Anthomyzidae & - & - & Current study \\
\hline Asilidae & Asilinae & Zosteria sp. & Current study \\
\hline Asteiidae & - & - & Current study \\
\hline Bibionidae & - & - & Current study \\
\hline Bombyliidae & - & - & Arabyat et al., 2004 \\
\hline \multirow[t]{3}{*}{ Calliphoridae } & Chrysomyinae & Chrysomya sp. & $\begin{array}{l}\text { Mazumdar et al., } \\
2010,2014\end{array}$ \\
\hline & Luciliinae & Hemipyrellia sp. & Current study \\
\hline & & Lucilia sp. & Islam et al., 2017 \\
\hline Canacidae & - & - & Mathis, 1992 \\
\hline
\end{tabular}


Table 1. Continued.

\begin{tabular}{|c|c|c|c|}
\hline Family & Subfamily & Scientific name & References \\
\hline Carnidae & - & - & Current study \\
\hline \multirow[t]{2}{*}{ Cecidomyiidae } & Cecidomyiinae & Contarinia maculipennis Felt, 1933 & Current study \\
\hline & & $\begin{array}{l}\text { Feltiella acarisuga (Vallot, 1827) } \\
\text { (Fig. 8) }\end{array}$ & Current study \\
\hline Celyphidae & & $\begin{array}{l}\text { Spaniocelyphus bigoti Karsch, } 1884 \\
\text { (Fig. 9) }\end{array}$ & Current study \\
\hline \multirow[t]{11}{*}{ Ceratopogonidae } & $\begin{array}{l}\text { Ceratopogonina } \\
\mathrm{e}\end{array}$ & $\begin{array}{l}\text { Culicoides actoni Smith, } 1929 \\
\text { (Fig. 10) }\end{array}$ & Current study \\
\hline & & Culicoides asiana Bellis, 2015 & Current study \\
\hline & & $\begin{array}{l}\text { Culicoides brevipalpis Delfinado, } \\
1961 \text { (Fig. 11) }\end{array}$ & Current study \\
\hline & & $\begin{array}{l}\text { Culicoides innoxius Sen and Das } \\
\text { Gupta, } 1959 \text { (Fig. 12) }\end{array}$ & Current study \\
\hline & & $\begin{array}{l}\text { Culicoides orientalis Macfie, } 1932 \\
\text { (Fig. 13) }\end{array}$ & Current study \\
\hline & & $\begin{array}{l}\text { Culicoides oxystoma Kieffer, } 1910 \\
\text { (Fig. 14) }\end{array}$ & Current study \\
\hline & & $\begin{array}{l}\text { Culicoides peregrines Kieffer, } 1910 \\
\text { (Fig. 15) }\end{array}$ & Current study \\
\hline & & $\begin{array}{l}\text { Culicoides shortti Smith and } \\
\text { Swaminathan, } 1932 \text { (Fig. 16) }\end{array}$ & Current study \\
\hline & & Culicoides similis Carter, 1920 (Fig. 17) & Current study \\
\hline & & $\begin{array}{l}\text { Culicoides sumatrae Macfie, } 1934 \\
\text { (Fig. 18) }\end{array}$ & Current study \\
\hline & Forcipomyiinae & Forcipomyia sp. & Current study \\
\hline \multirow[t]{12}{*}{ Chironomidae } & Chironominae & $\begin{array}{l}\text { Chironomus circumdatus (Kieffer, } \\
\text { 1916) (Fig. 19) }\end{array}$ & Current study \\
\hline & & $\begin{array}{l}\text { Chironomus crassiforceps } \\
\text { (Kieffer, 1916) (Fig. 20) }\end{array}$ & Current study \\
\hline & & $\begin{array}{l}\text { Chironomus javanus Kieffer, } 1924 \\
\text { (Fig. 21) }\end{array}$ & Current study \\
\hline & & Cladotanytarsus sp. & Current study \\
\hline & & $\begin{array}{l}\text { Kiefferulus calligaster (Kieffer, } \\
\text { 1911) (Fig. 22) }\end{array}$ & Current study \\
\hline & & $\begin{array}{l}\text { Microchironomus cf. tener (Kieffer, } \\
\text { 1918) (Fig. 23) }\end{array}$ & Current study \\
\hline & & $\begin{array}{l}\text { Polypedilum cf. allansoni Freeman, } \\
1958 \text { (Fig. 24) }\end{array}$ & Current study \\
\hline & & $\begin{array}{l}\text { Tanytarsus formosanus Kieffer,1912 } \\
\text { (Fig. 25) }\end{array}$ & Current study \\
\hline & & Zavreliella sp. & Current study \\
\hline & Tanypodinae & Denopelopia sp. & Current study \\
\hline & & Paramerina sp. & Current study \\
\hline & & Procladius sp. & Current study \\
\hline
\end{tabular}


Table 1. Continued.

\begin{tabular}{|c|c|c|c|}
\hline Family & Subfamily & Scientific name & References \\
\hline \multirow[t]{6}{*}{ Chloropidae } & Chloropinae & Pachylophus sp. & Catling \& Islam, 2013 \\
\hline & Oscinellinae & Anatrichus sp. & Catling \& Islam, 2013 \\
\hline & & Cadrema sp. & Current study \\
\hline & & Oscinella sp. & Current study \\
\hline & Rhodesiellinae & Neorhodesiella sp. & Current study \\
\hline & & Rhodesiella sp. & Current study \\
\hline Chyromyidae & & - & Current study \\
\hline Clusiidae & & - & Current study \\
\hline Cryptochetidae & & - & Current study \\
\hline \multirow[t]{13}{*}{ Culicidae } & Anophelinae & $\begin{array}{l}\text { Anopheles vagus Donitz, } 1902 \\
\text { (Fig. 26) }\end{array}$ & $\begin{array}{l}\text { Huda \& Majumder, } \\
\text { 1985; Irish et al., } 2016\end{array}$ \\
\hline & Culicinae & $\begin{array}{l}\text { Aedes albopictus (Skuse,1894) } \\
\text { (Fig. 27) }\end{array}$ & $\begin{array}{l}\text { Huda \& Majumder, } \\
\text { 1985; Irish et al., } 2016\end{array}$ \\
\hline & & $\begin{array}{l}\text { Armigeres subalbatus (Coquillett, } \\
\text { 1898) (Fig. 28) }\end{array}$ & Irish et al., 2016 \\
\hline & & $\begin{array}{l}\text { Culex bitaeniorhynchus Giles, } 1901 \\
\text { (Fig. 29) }\end{array}$ & Irish et al., 2016 \\
\hline & & $\begin{array}{l}\text { Culex fuscocephala Theobald, } \\
1907 \text { (Fig. 30) }\end{array}$ & Irish et al., 2016 \\
\hline & & $\begin{array}{l}\text { Culex gelidus Theobald, } 1901 \\
\text { (Fig. 31) }\end{array}$ & Irish et al., 2016 \\
\hline & & $\begin{array}{l}\text { Culex infantulus Edwards, } 1922 \\
\text { (Fig. 32) }\end{array}$ & Current study \\
\hline & & $\begin{array}{l}\text { Culex pallidothorax Theobald, } 1905 \\
\text { (Fig. 33) }\end{array}$ & Irish et al., 2016 \\
\hline & & $\begin{array}{l}\text { Culex pipiens Linnaeus, } 1758 \\
\text { (Fig. 34) }\end{array}$ & $\begin{array}{l}\text { Ameen \& } \\
\text { Moizuddin, } 1975\end{array}$ \\
\hline & & $\begin{array}{l}\text { Culex rubithoracis (Leicester, 1908) } \\
\text { (Fig. 35) }\end{array}$ & Current study \\
\hline & & $\begin{array}{l}\text { Culex tritaeniorhynchus Giles, } 1901 \\
\text { (Fig. 36) }\end{array}$ & $\begin{array}{l}\text { Huda \& Majumder, } \\
\text { 1985; Irish et al., } 2016\end{array}$ \\
\hline & & $\begin{array}{l}\text { Culex vishnui Theobald, } 1901 \\
\text { (Fig. 37) }\end{array}$ & $\begin{array}{l}\text { Huda \& Majumder, } \\
\text { 1985; Irish et al., } 2016\end{array}$ \\
\hline & & Lutzia sp. & Irish et al., 2016 \\
\hline \multirow[t]{4}{*}{ Dolichopodidae } & Diaphorinae & Chrysotus sp. & Current study \\
\hline & Dolichopodinae & $\begin{array}{l}\text { Paraclius digitatus Zhang, Yang \& } \\
\text { Grootaert, } 2007 \text { (Fig. 38) }\end{array}$ & Current study \\
\hline & Hydrophorinae & Thinophilus sp. & Current study \\
\hline & Medeterinae & $\begin{array}{l}\text { Medetera grisescens de Meijere, } \\
1916 \text { (Fig. 39) }\end{array}$ & Current study \\
\hline Drosophilidae & Drosophilinae & $\begin{array}{l}\text { Drosophila albomicans (Duda, 1923) } \\
\text { (Fig. 40) }\end{array}$ & Current study \\
\hline
\end{tabular}


Table 1. Continued.

\begin{tabular}{|c|c|c|c|}
\hline Family & Subfamily & Scientific name & References \\
\hline \multirow[t]{7}{*}{ Drosophilidae } & Drosophilinae & $\begin{array}{l}\text { Drosophila sulfurigaster (Duda, } \\
\text { 1923) (Fig. 41) }\end{array}$ & Current study \\
\hline & & $\begin{array}{l}\text { Mycodrosophila aqua Bock, } 1980 \\
\text { (Fig. 42) }\end{array}$ & Current study \\
\hline & & $\begin{array}{l}\text { Scaptodrosophila riverata (Singh \& } \\
\text { Gupta, 1977) }\end{array}$ & Current study \\
\hline & & $\begin{array}{l}\text { Scaptomyza pallid (Zetterstedt, } \\
\text { 1847) (Fig. 43) }\end{array}$ & Current study \\
\hline & Steganinae & Leucophenga angusta Okada, 1956 & Current study \\
\hline & & $\begin{array}{l}\text { Leucophenga taiwanensis Lin \& } \\
\text { Wheeler, } 1972\end{array}$ & Current study \\
\hline & & $\begin{array}{l}\text { Stegana kanmiyai Okada \& } \\
\text { Sidorenko, } 1992 \text { (Fig. 44) }\end{array}$ & Current study \\
\hline Empididae & - & - & Current study \\
\hline \multirow[t]{7}{*}{ Ephydridae } & Ephydrinae & Brachydeutera sp. & Current study \\
\hline & & Scatella sp. & Current study \\
\hline & Ilytheinae & Nostima sp. & Current study \\
\hline & Notiphilinae & $\begin{array}{l}\text { Hydrellia flaviceps (Meigen, 1830) } \\
\text { (Fig. 45) }\end{array}$ & Current study \\
\hline & & $\begin{array}{l}\text { Hydrellia pakistanae Deonier, } 1978 \\
\text { (Fig. 46) }\end{array}$ & Current study \\
\hline & Psilopinae & Polytrichophora sp. & Current study \\
\hline & & Psilopa sp. & Current study \\
\hline Hippoboscidae & - & - & Current study \\
\hline Hybotidae & Tachydromiinae & - & Current study \\
\hline Keroplatidae & - & - & Current study \\
\hline Lauxaniidae & - & - & Current study \\
\hline \multirow[t]{2}{*}{ Limoniidae } & Limoniinae & Orimarga sp. & Current study \\
\hline & & Trentepohlia sp. & Current study \\
\hline Lonchaeidae & Lonchaeinae & Lamprolonchaea sp. & Current study \\
\hline Micropezidae & & - & $\begin{array}{l}\text { Jui et al., 2007; Hill, } \\
2008\end{array}$ \\
\hline \multirow[t]{3}{*}{ Milichiidae } & Madizinae & $\begin{array}{l}\text { Desmometopa sordida (Fallén,1820) } \\
\text { (Fig. 47) }\end{array}$ & Current study \\
\hline & & $\begin{array}{l}\text { Desmometopa varipalpis Malloch, } \\
1927 \text { (Fig. 48) }\end{array}$ & Current study \\
\hline & Milichiinae & Milichiella sp. & Current study \\
\hline \multirow[t]{4}{*}{ Muscidae } & Coenosiinae & Coenosia attenuata Stein, 1903 (Fig. 49) & Current study \\
\hline & & Limnophora sp. & Current study \\
\hline & & $\begin{array}{l}\text { Lispe assimilis Wiedemann, } 1824 \\
\text { (Fig. 50) }\end{array}$ & Current study \\
\hline & & $\begin{array}{l}\text { Lispe nicobarensis Schiner, } 1868 \\
\text { (Fig. 51) }\end{array}$ & Current study \\
\hline
\end{tabular}


Table 1. Continued.

\begin{tabular}{|c|c|c|c|}
\hline Family & Subfamily & Scientific name & References \\
\hline \multirow[t]{17}{*}{ Muscidae } & Coenosiinae & $\begin{array}{l}\text { Lispe sericipalpis (Stein, 1904) } \\
\text { (Fig. 52) }\end{array}$ & Current study \\
\hline & & $\begin{array}{l}\text { Pygophora respondens (Walker, } \\
\text { 1859) (Fig. 53) }\end{array}$ & Current study \\
\hline & Muscinae & $\begin{array}{l}\text { Haematobia irritans (Linnaeus, } \\
\text { 1758) (Fig. 54) }\end{array}$ & Current study \\
\hline & & $\begin{array}{l}\text { Haematobosca sanguinoleant } \\
\text { (Austen, 1909) (Fig. 55) }\end{array}$ & Current study \\
\hline & & Morellia sp. & Current study \\
\hline & & $\begin{array}{l}\text { Musca cnofiscate Speiser, } 1924 \\
\text { (Fig. 56) }\end{array}$ & Current study \\
\hline & & $\begin{array}{l}\text { Musca convexifrons Thomson, } 1869 \\
\text { (Fig. 57) }\end{array}$ & Current study \\
\hline & & $\begin{array}{l}\text { Musca crassirostris Stein, } 1903 \\
\text { (Fig. 58) }\end{array}$ & Current study \\
\hline & & $\begin{array}{l}\text { Musca domestica Linnaeus, } 1758 \\
\text { (Fig. 59) }\end{array}$ & $\begin{array}{l}\text { Mazumdar et al., } \\
2010\end{array}$ \\
\hline & & Musca formosana Malloch,1910 & Current study \\
\hline & & $\begin{array}{l}\text { Musca ventrosa Wiedemann, } 1830 \\
\text { (Fig. 60) }\end{array}$ & Current study \\
\hline & & $\begin{array}{l}\text { Neomyia timorensis (Robineau- } \\
\text { Desvoidy, 1830) (Fig. 61) }\end{array}$ & Current study \\
\hline & & $\begin{array}{l}\text { Stomoxys calcitrans } \\
\text { (Linnaeus, 1758) (Fig. 62) }\end{array}$ & EBO, 2015 \\
\hline & & $\begin{array}{l}\text { Stomoxys indicus Picard, } 1908 \\
\text { (Fig. 63) }\end{array}$ & Current study \\
\hline & & $\begin{array}{l}\text { Stomoxys uruma Shinonaga \& } \\
\text { Kano, } 1966 \text { (Fig. 64) }\end{array}$ & Current study \\
\hline & Phaoniinae & $\begin{array}{l}\text { Atherigona orientalis Schiner, } 1868 \\
\text { (Fig. } 65 \text { ) }\end{array}$ & Current study \\
\hline & & $\begin{array}{l}\text { Atherigona oryzae Malloch, } 1925 \\
\text { (Fig. 66) }\end{array}$ & Catling \& Islam, 2013 \\
\hline Mycetophilidae & & - & Current study \\
\hline Mythicomyiidae & & - & Current study \\
\hline Neriidae & & - & Current study \\
\hline Odiniidae & & - & Current study \\
\hline Periscelididae & & - & Current study \\
\hline \multirow[t]{3}{*}{ Phoridae } & Metopininae & Megaselia sp. & Alam et al., 2015 \\
\hline & Phorinae & Diplonevra sp. & Current study \\
\hline & & $\begin{array}{l}\text { Dohrniphora cornuta (Bigot, 1857) } \\
\text { (Fig. 67) }\end{array}$ & Current study \\
\hline \multirow[t]{2}{*}{ Pipunculidae } & Pipunculinae & Eudorylas sp. & Current study \\
\hline & & Tomosvaryella sp. & Current study \\
\hline Platypezidae & & - & Current study \\
\hline
\end{tabular}


Table 1. Continued.

\begin{tabular}{|c|c|c|c|}
\hline Family & Subfamily & Scientific name & References \\
\hline Platystomatidae & & - & Current study \\
\hline Psilidae & & - & Current study \\
\hline \multirow[t]{2}{*}{ Psychodidae } & Psychodinae & Brunettia sp. & Current study \\
\hline & & Psychoda alternate Say, 1824 (Fig. 68) & Current study \\
\hline Rhiniidae & Rhiniinae & $\begin{array}{l}\text { Stomorhina discolor (Fabricius, } \\
\text { 1794) (Fig. 69) }\end{array}$ & Sitompul et al., 2018 \\
\hline Rhinophoridae & - & - & Current study \\
\hline \multirow[t]{5}{*}{ Sarcophagidae } & Sarcophaginae & $\begin{array}{l}\text { Sarcophaga albiceps Meigen, } 1826 \\
\text { (Fig. 70) }\end{array}$ & Sharma et al., 2017 \\
\hline & & $\begin{array}{l}\text { Sarcophaga misera Walker, } 1849 \\
\text { (Fig. 71) }\end{array}$ & $\begin{array}{l}\text { Pape, 1996; SMT, } \\
2020\end{array}$ \\
\hline & & $\begin{array}{l}\text { Sarcophaga princeps Wiedemann, } \\
1830 \text { (Fig. 72) }\end{array}$ & Current study \\
\hline & & $\begin{array}{l}\text { Sarcophaga scopariiformis Senior- } \\
\text { White, } 1927 \text { (Fig. 73) }\end{array}$ & Current study \\
\hline & & $\begin{array}{l}\text { Sarcophaga taenionota } \\
\text { (Wiedemann, 1819) (Fig. 74) }\end{array}$ & Pape, 1996 \\
\hline Scatopsidae & - & - & Current study \\
\hline \multirow[t]{4}{*}{ Sciaridae } & & $\begin{array}{l}\text { Bradysia ocellaris (Comstock, 1882) } \\
\text { (Fig. 75) }\end{array}$ & Current study \\
\hline & & Cosmosciara sp. & Current study \\
\hline & & Hyperlasion sp. & Current study \\
\hline & & Sciara sp. & Current study \\
\hline \multirow[t]{6}{*}{ Sepsidae } & Sepsinae & $\begin{array}{l}\text { Australosepsis frontalis (Walker, } \\
\text { 1860) (Fig. 76) }\end{array}$ & Current study \\
\hline & & Dicranosepsis cf. sauteri Ozerov, 2003 & Current study \\
\hline & & $\begin{array}{l}\text { Dicranosepsis crinite (Duda, 1926) } \\
\text { (Fig. 77) }\end{array}$ & Current study \\
\hline & & $\begin{array}{l}\text { Sepsis dissimilis Brunetti, } 1909 \\
\text { (Fig. 78) }\end{array}$ & Current study \\
\hline & & $\begin{array}{l}\text { Sepsis nitens Wiedemann, } 1824 \\
\text { (Fig. } 79 \text { ) }\end{array}$ & Current study \\
\hline & Toxopodinae & Toxopoda sp. & Iwasa et al., 1991 \\
\hline Simuliidae & - & - & Current study \\
\hline \multirow[t]{5}{*}{ Sphaeroceridae } & Limosininae & $\begin{array}{l}\text { Coproica ferruginata (Stenhammar, } \\
\text { 1855) (Fig. 80) }\end{array}$ & Current study \\
\hline & & $\begin{array}{l}\text { Coproica hirtula (Rondani, } \\
\text { 1880) (Fig. 81) }\end{array}$ & Current study \\
\hline & & Rachispoda sp. & Current study \\
\hline & & $\begin{array}{l}\text { Spelobia bifrons (Stenhammar, } \\
\text { 1855) (Fig. 82) }\end{array}$ & Current study \\
\hline & & $\begin{array}{l}\text { Trachyopella lineafrons (Spuler, } \\
\text { 1925) (Fig. 83) }\end{array}$ & Current study \\
\hline
\end{tabular}


Table 1. Continued.

\begin{tabular}{|c|c|c|c|}
\hline Family & Subfamily & Scientific name & References \\
\hline Sphaeroceridae & Limosininae & $\begin{array}{l}\text { Trachyopella straminea Rohacek \& } \\
\text { Marshall, } 1986 \text { (Fig. 84) }\end{array}$ & Current study \\
\hline \multirow[t]{8}{*}{ Stratiomyidae } & & - & Current study \\
\hline & Clitellariinae & - & Current study \\
\hline & & Octorthra sp. & Current study \\
\hline & Hermetiinae & $\begin{array}{l}\text { Hermetia illucens (Linnaeus, 1758) } \\
\text { (Fig. 85) }\end{array}$ & Current study \\
\hline & Pachygastrinae & - & Current study \\
\hline & Sarginae & $\begin{array}{l}\text { Microchrysa flaviventris } \\
\text { (Wiedemann, 1824) (Fig. 86) }\end{array}$ & Current study \\
\hline & Stratiomyinae & Caloparyphus sp. & Current study \\
\hline & Eristalinae & $\begin{array}{l}\text { Syritta orientalis Macquart, } 1842 \\
\text { (Fig. } 87 \text { ) }\end{array}$ & Current study \\
\hline \multirow[t]{6}{*}{ Syrphidae } & Syrphinae & $\begin{array}{l}\text { Allobaccha amphithoe (Walker, } \\
\text { 1849) (Fig. 88) }\end{array}$ & Current study \\
\hline & & $\begin{array}{l}\text { Allograpta medanensis (Meijere, } \\
\text { 1914) (Fig. 89) }\end{array}$ & Current study \\
\hline & & Melanostoma sp. & Current study \\
\hline & & Paragus sp. & Mazumdar et al., 2014 \\
\hline & & $\begin{array}{l}\text { Simosyrphus scutellaris (Fabricius, } \\
\text { 1805) (Fig. 90) }\end{array}$ & Current study \\
\hline & & Sphaerophoria sp. & EBO, 2014 \\
\hline \multirow[t]{3}{*}{ Tabanidae } & Tabaninae & $\begin{array}{l}\text { Atylotus agrestis (Wiedemann, 1828) } \\
\text { (Fig. 91) }\end{array}$ & Current study \\
\hline & & $\begin{array}{l}\text { Tabanus rubidus Wiedemann, } 1821 \\
\text { (Fig. 92) }\end{array}$ & Alfred et al., 1999 \\
\hline & & $\begin{array}{l}\text { Tabanus striatus Fabricius, } 1787 \\
\text { (Fig. 93) }\end{array}$ & $\begin{array}{l}\text { Alfred et al., 1999; } \\
\text { Maity et al., } 2015\end{array}$ \\
\hline \multirow[t]{3}{*}{ Tachinidae } & Exoristinae & $\begin{array}{l}\text { Aplomya metallica (Wiedemann, } \\
\text { 1824) (Fig. 94) }\end{array}$ & Islam, 2015 \\
\hline & & $\begin{array}{l}\text { Exorista xanthaspis (Wiedemann, } \\
\text { 1830) (Fig. 95) }\end{array}$ & Islam, 2015 \\
\hline & Phasiinae & Gymnosoma sp. & Current study \\
\hline \multirow[t]{3}{*}{ Tephritidae } & Dacinae & $\begin{array}{l}\text { Bactrocera cucurbitae (Coquillett, } \\
\text { 1899) (Fig. 96) }\end{array}$ & $\begin{array}{l}\text { Akhtaruzzaman et } \\
\text { al., 1999; Leblanc et } \\
\text { al., 2013; Rahman et } \\
\text { al., } 2019\end{array}$ \\
\hline & & $\begin{array}{l}\text { Bactrocera diversa (Coquillett, } \\
\text { 1904) (Fig. 97) }\end{array}$ & Leblanc et al., 2013 \\
\hline & & $\begin{array}{l}\text { Dacus longicornis Wiedemann, } \\
1830 \text { (Fig. 98) }\end{array}$ & $\begin{array}{l}\text { Khan, 2009; Leblanc } \\
\text { et al., } 2013\end{array}$ \\
\hline Therevidae & - & - & Current study \\
\hline Ulidiidae & Ulidiinae & Physiphora sp. (Fig. 99) & Current study \\
\hline Xylomyidae & - & - & Current study \\
\hline
\end{tabular}



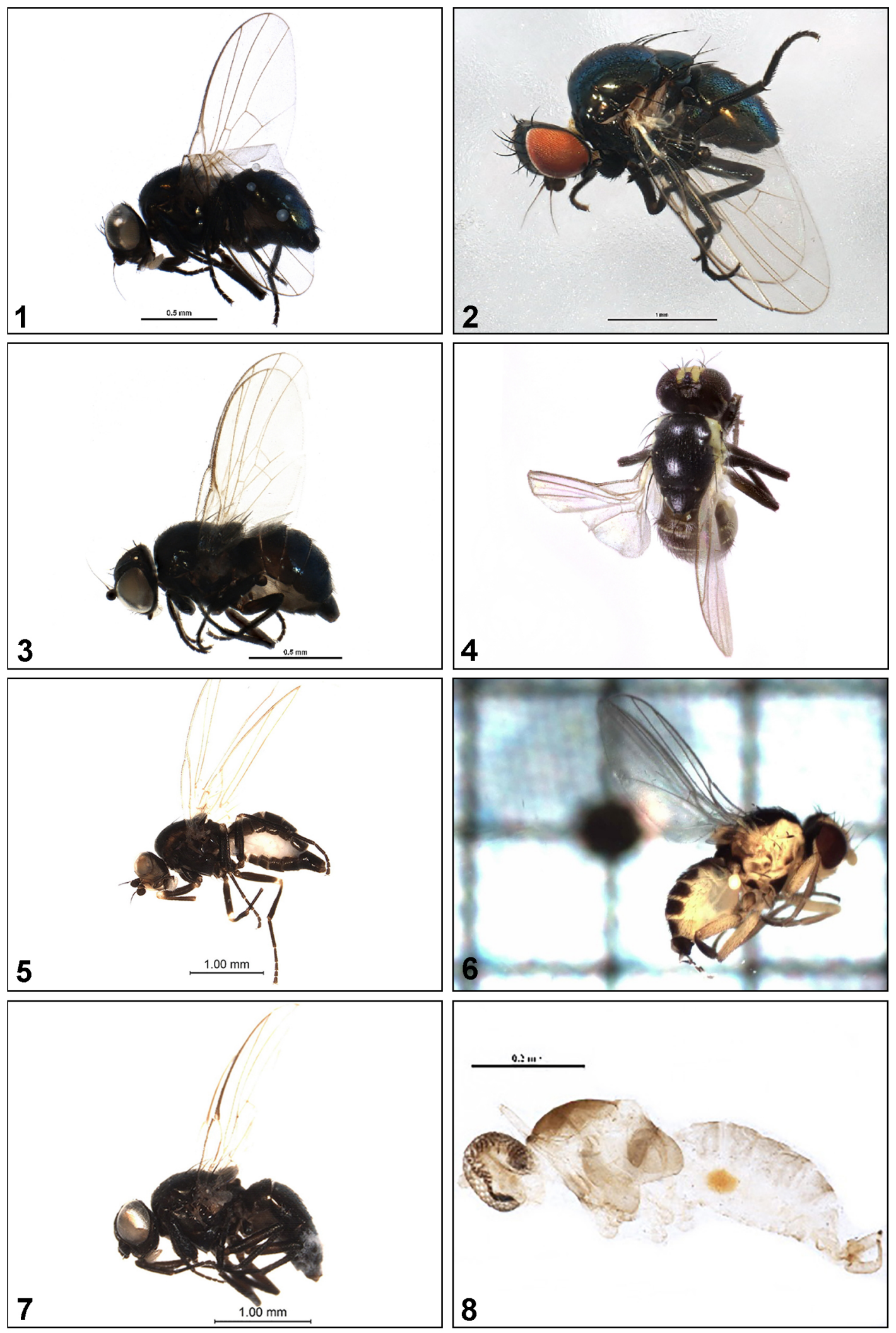

Figures 1-8. General habitus of True flies collected in Bangladesh. 1. Melanagromyza cleomae; 2. Melanagromyza obtusa; 3. Ophiomyia phaseoli; 4. Calycomyza lantanae; 5. Chromatomyia horticola; 6. Liriomyza sativae; 7. Phytomyza thalictrella; 8. Feltiella acarisuga. 

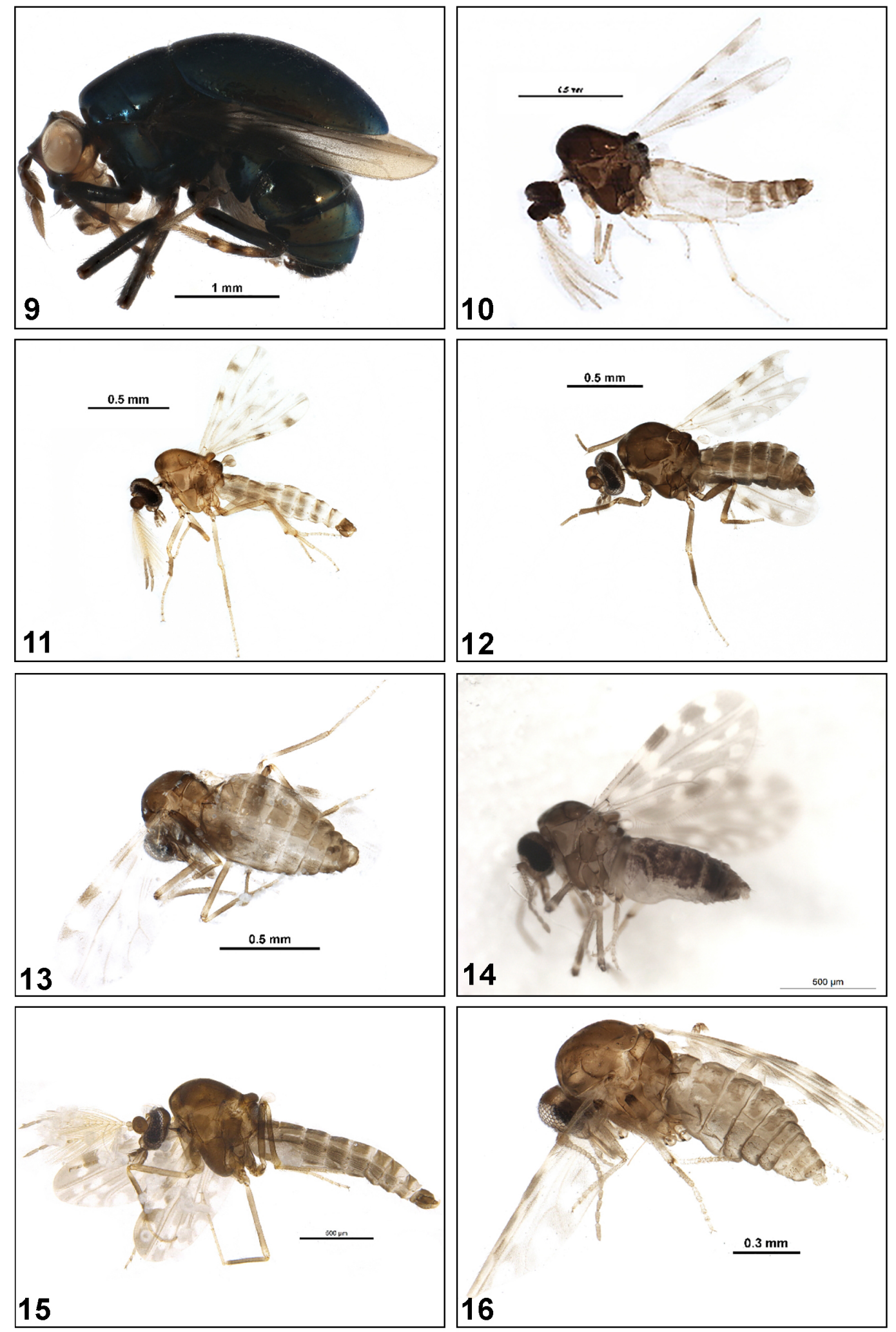

Figures 9-16. General habitus of True flies collected in Bangladesh. 9. Spaniocelyphus bigoti; 10. Culicoides actoni; 11. Culicoides brevipalpis; 12. Culicoides innoxius; 13. Culicoides orientalis; 14. Culicoides oxystoma; 15. Culicoides peregrines; 16. Culicoides shortti. 

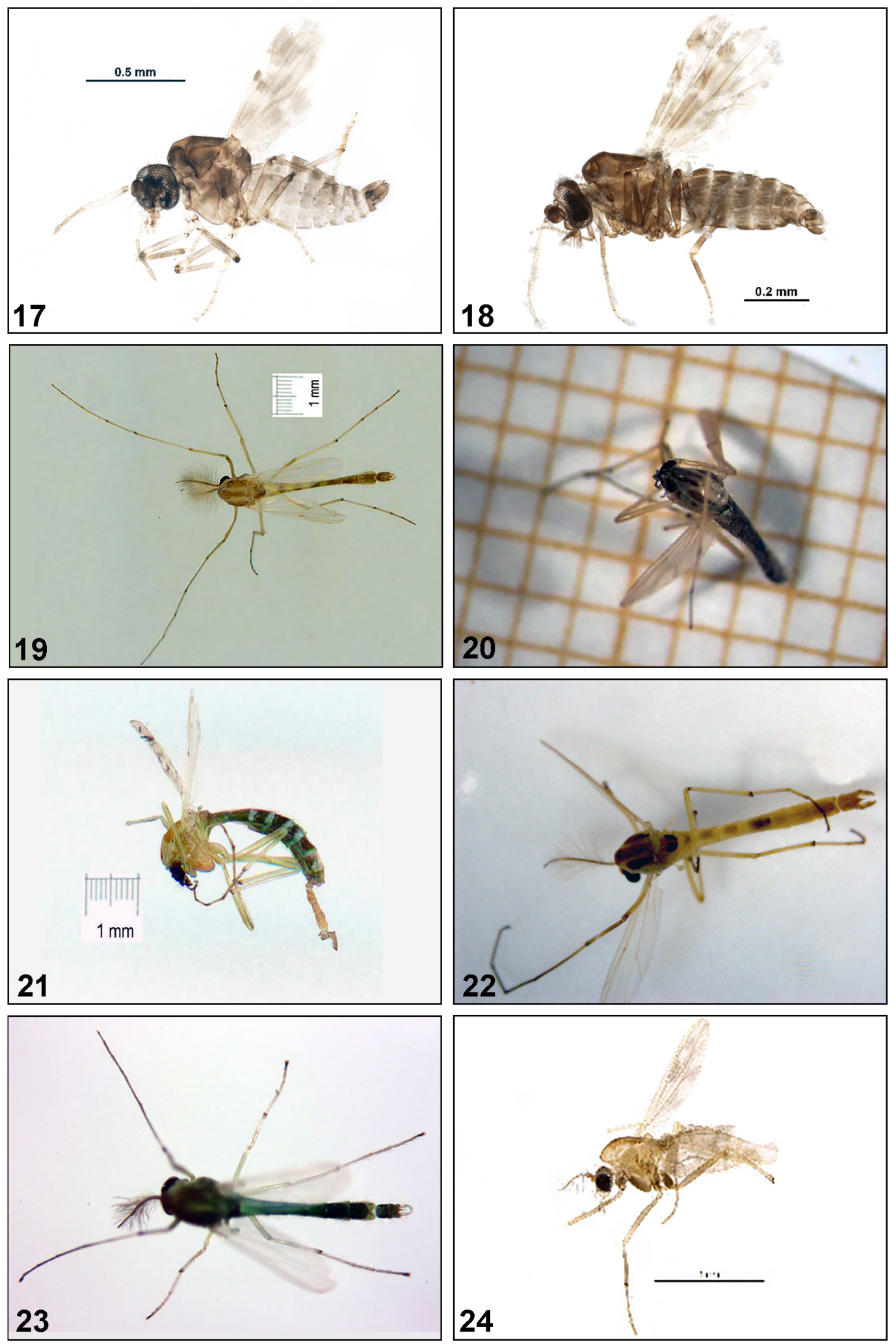

Figures 17-24. General habitus of True flies collected in Bangladesh. 17. Culicoides similis; 18. Culicoides sumatrae; 19. Chironomus circumdatus; 20. Chironomus crassiforceps; 21. Chironomus javanus; 22. Kiefferulus calligaster; 23. Microchironomus cf. tener; 24. Polypedilum cf. allansoni. 

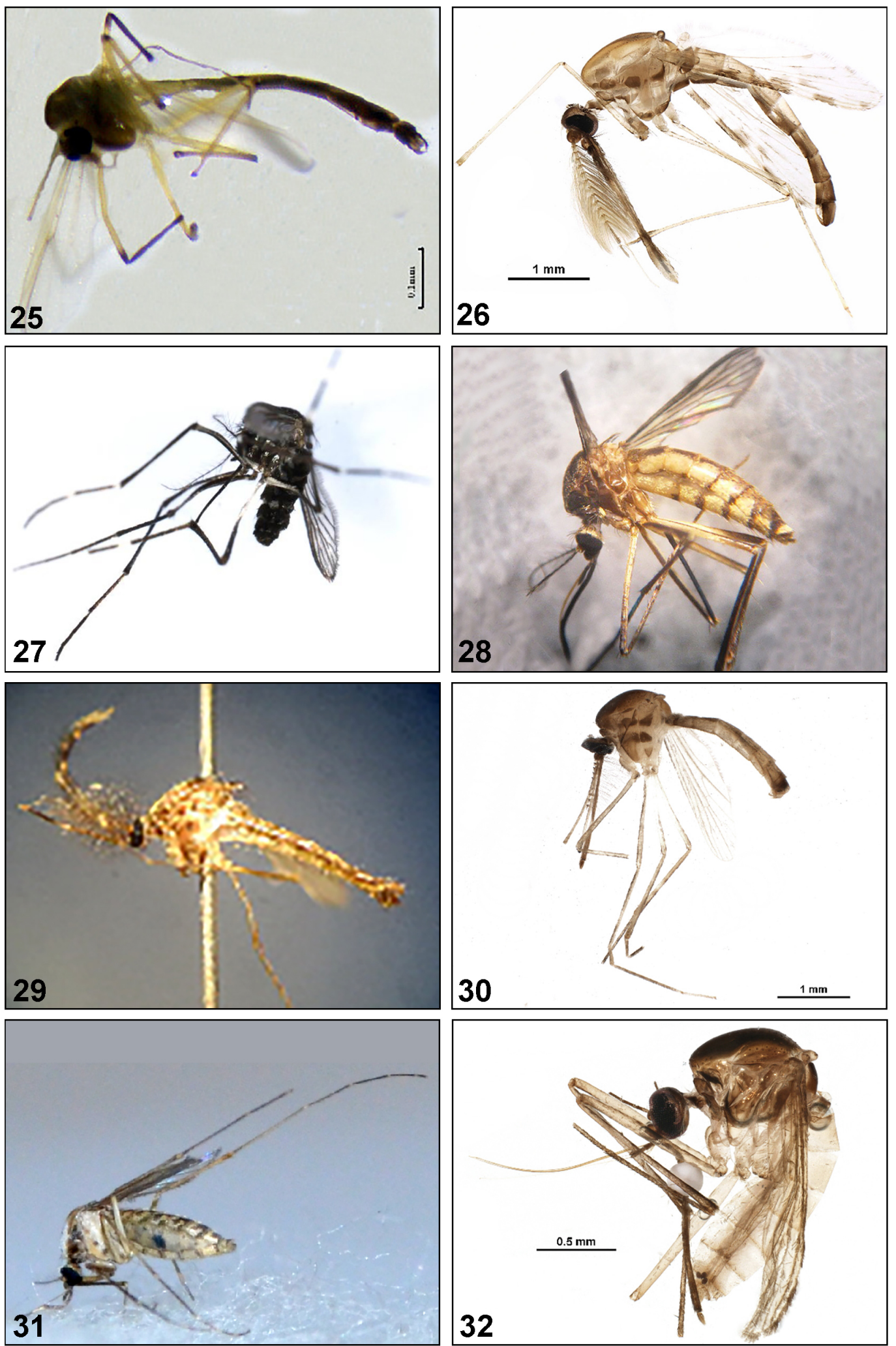

Figures 25-32. General habitus of True flies collected in Bangladesh. 25. Tanytarsus formosanus; 26. Anopheles vagus; 27. Aedes albopictus; 28. Armigeres subalbatus; 29. Culex bitaeniorhynchus; 30. Culex fuscocephala; 31. Culex gelidus; 32. Culex infantulus. 

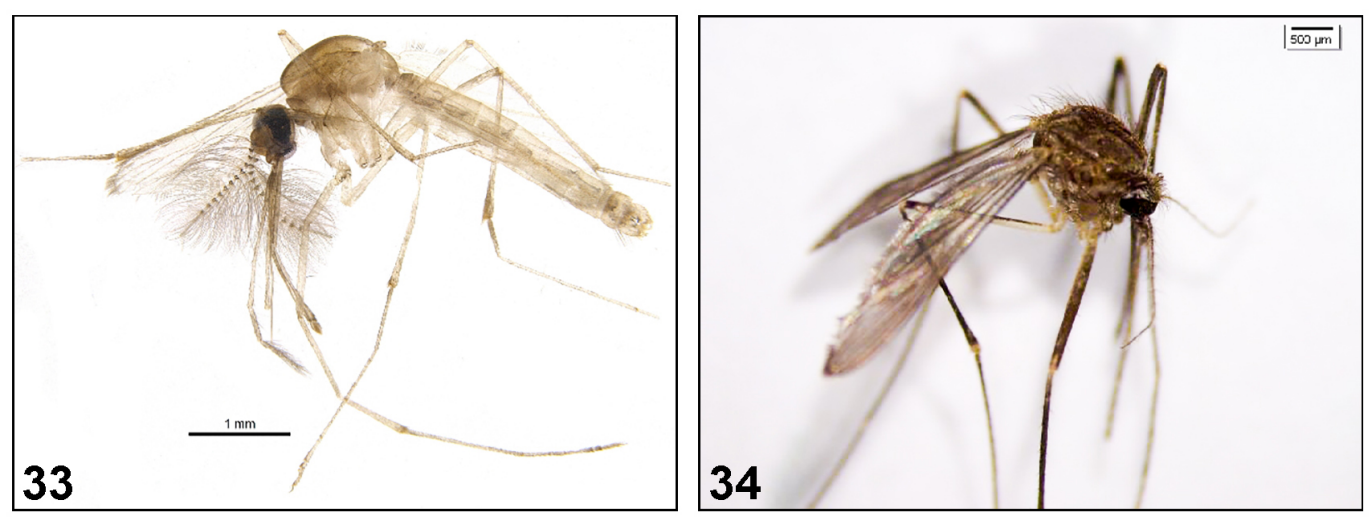

35
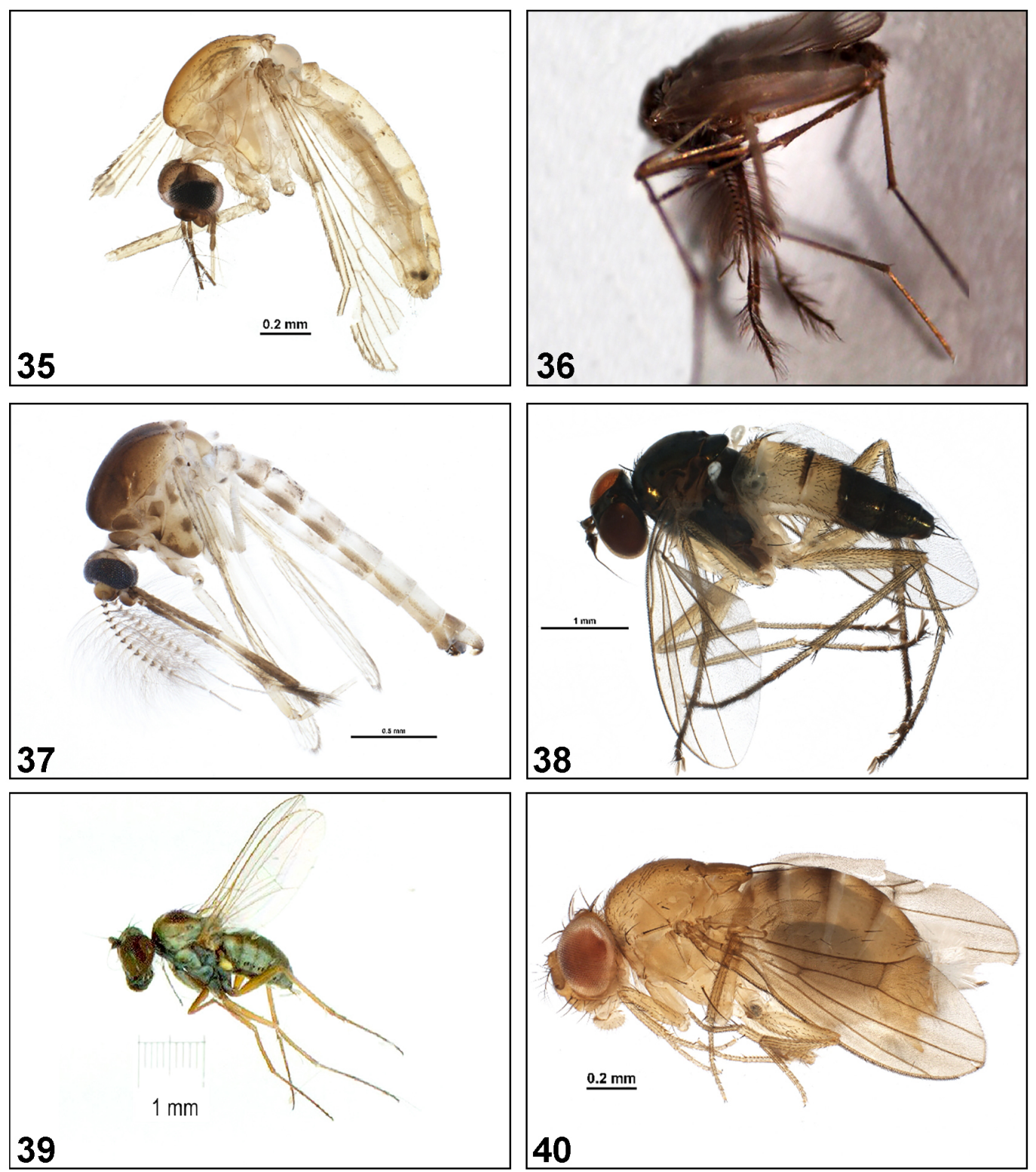

Figures 33-40. General habitus of True flies collected in Bangladesh. 33. Culex pallidothorax; 34. Culex pipiens; 35. Culex rubithoracis; 36. Culex tritaeniorhynchus; 37. Culex vishnui; 38. Paraclius digitatus; 39. Medetera grisescens; 40. Drosophila albomicans. 

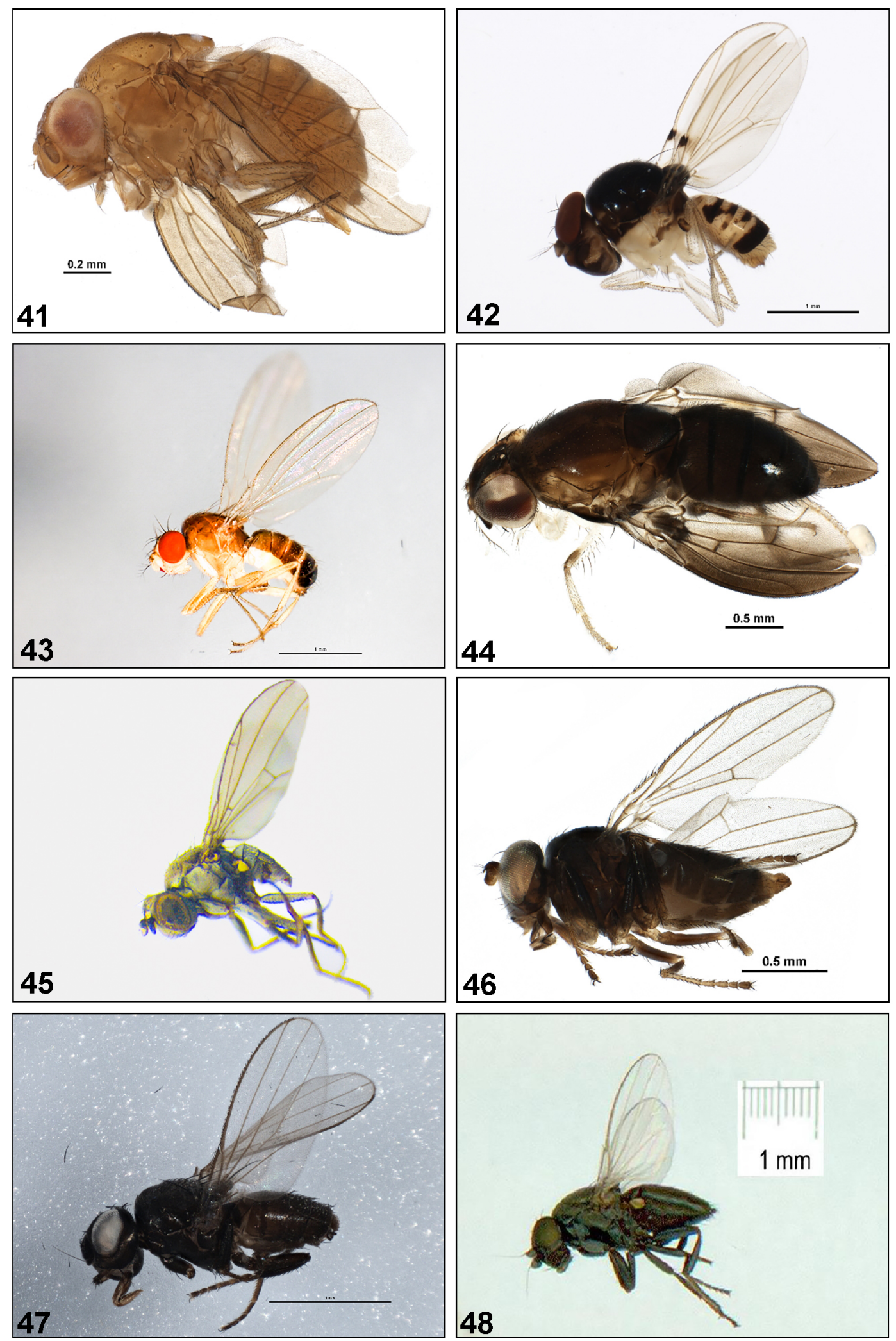

Figures 41-48. General habitus of True flies collected in Bangladesh. 41. Drosophila sulfurigaster; 42. Mycodrosophila aqua; 43. Scaptomyza pallid; 44. Stegana kanmiyai; 45. Hydrellia flaviceps; 46. Hydrellia pakistanae; 47. Desmometopa sordida; 48. Desmometopa varipalpis. 

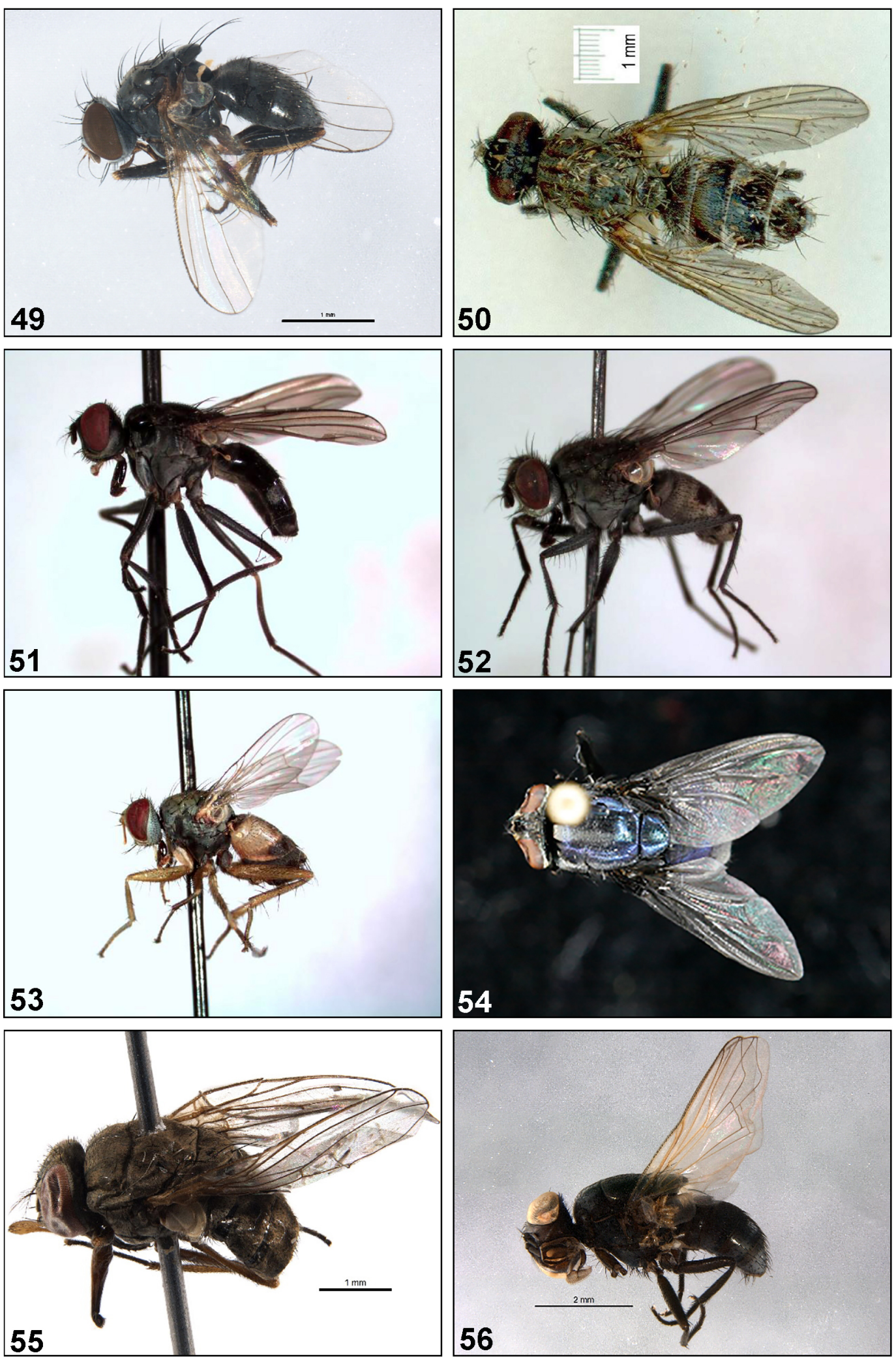

Figures 49-56. General habitus of True flies collected in Bangladesh. 49. Coenosia attenuata; 50. Lispe assimilis; 51. Lispe nicobarensis; 52. Lispe sericipalpis; 53. Pygophora respondens; 54. Haematobia irritans; 55. Haematobosca sanguinoleanta; 56. Musca cnofiscate. 

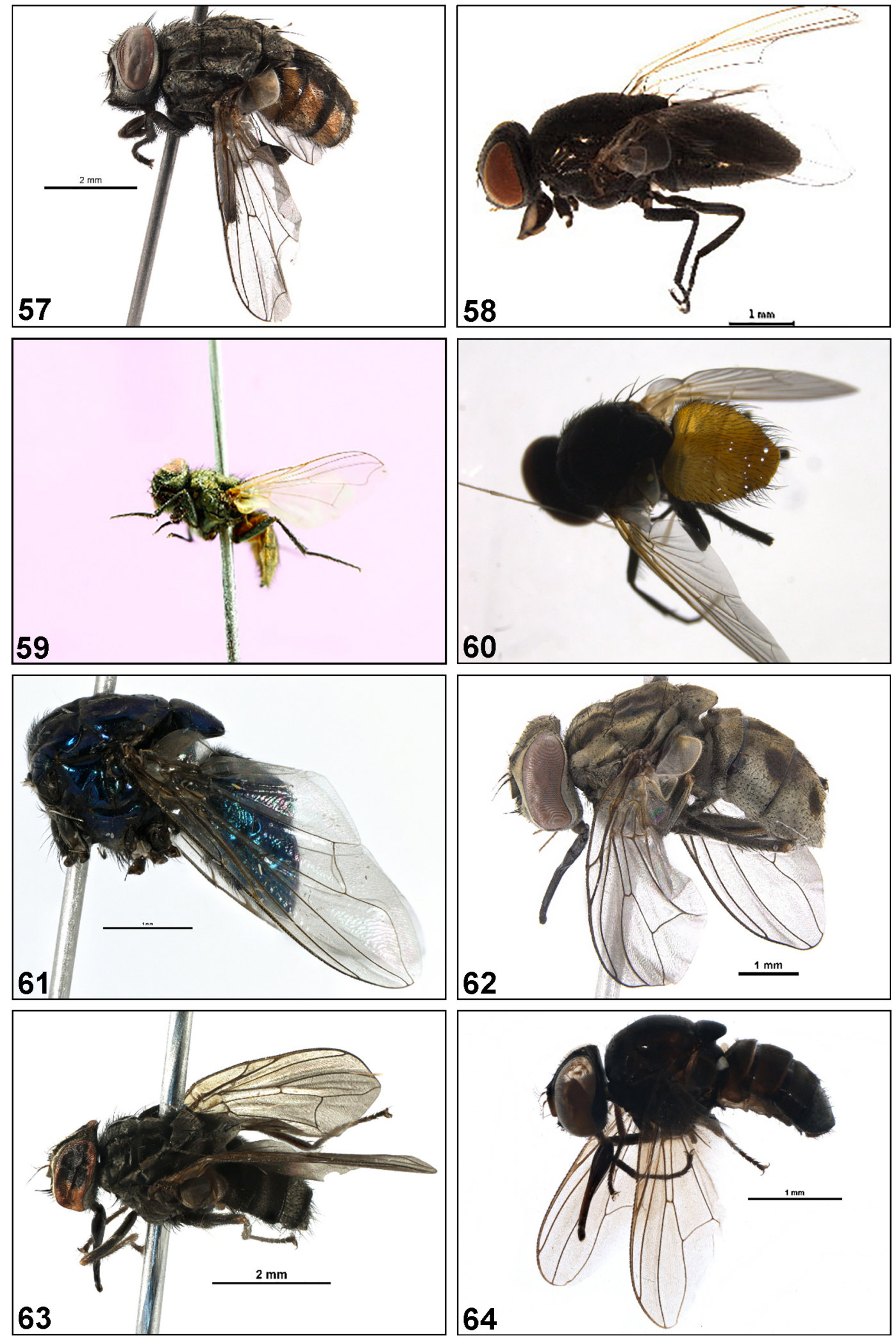

Figures 57-64. General habitus of True flies collected in Bangladesh. 57. Musca convexifrons; 58. Musca crassirostris; 59. Musca domestica; 60. Musca ventrosa; 61. Neomyia timorensis; 62. Stomoxys calcitrans; 63. Stomoxys indicus; 64. Stomoxys uruma. 

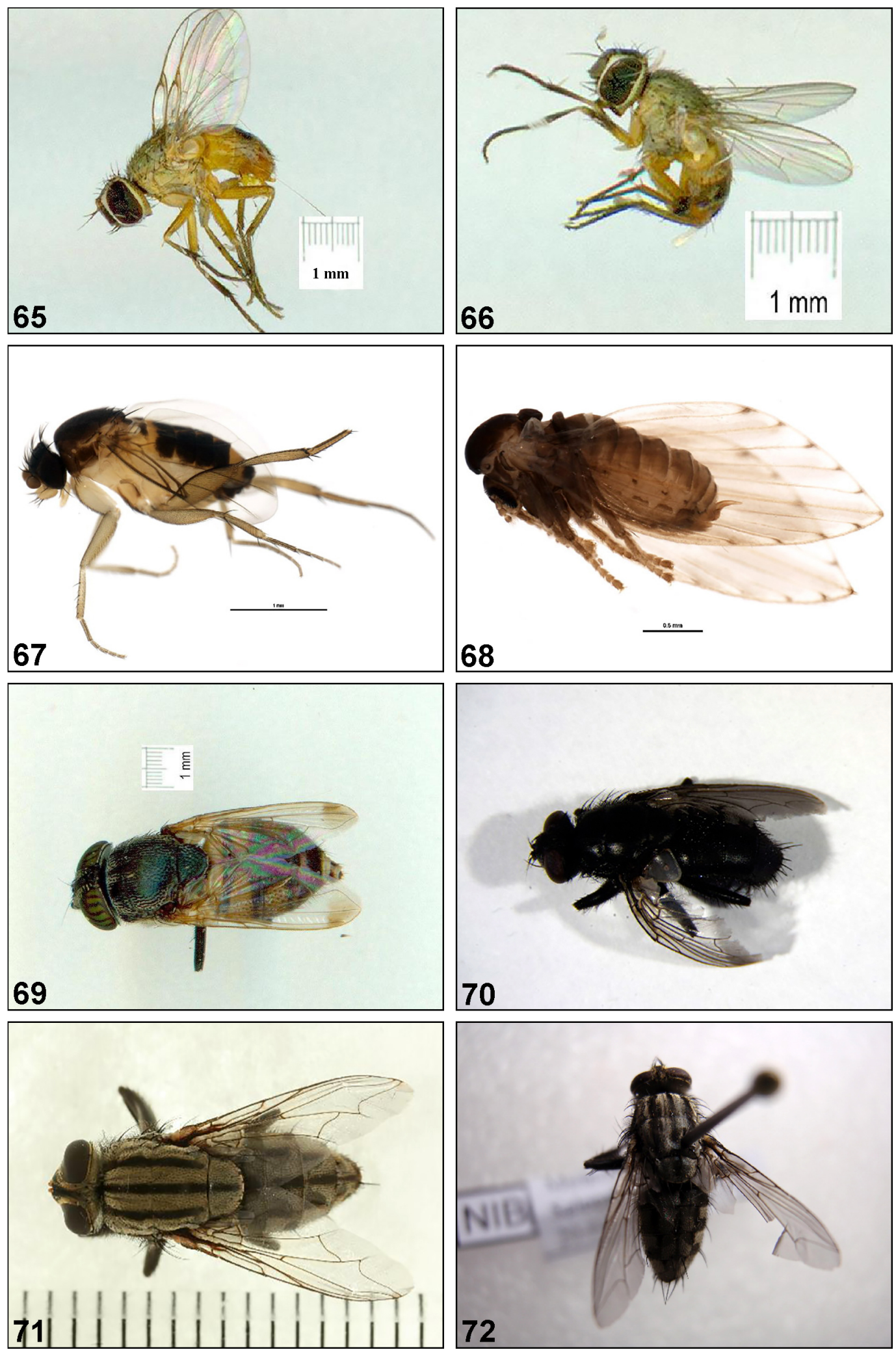

Figures 65-72. General habitus of True flies collected in Bangladesh. 65. Atherigona orientalis; 66. Atherigona oryzae; 67. Dohrniphora cornuta; 68. Psychoda alternate; 69. Stomorhina discolor; 70. Sarcophaga albiceps; 71. Sarcophaga misera; 72. Sarcophaga princeps. 

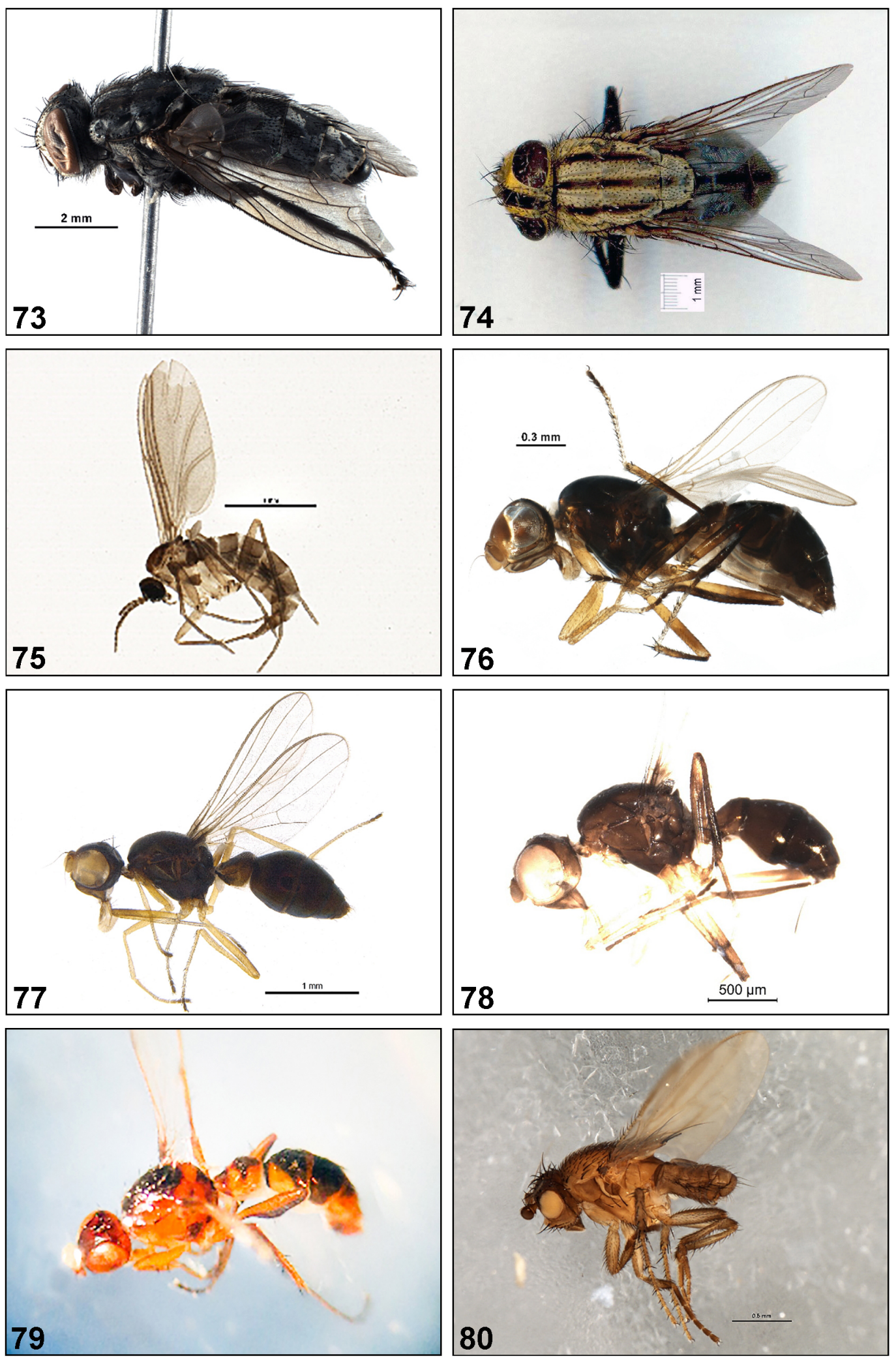

Figures 73-80. General habitus of True flies collected in Bangladesh. 73. Sarcophaga scopariiformis; 74. Sarcophaga taenionota; 75. Bradysia ocellaris; 76. Australosepsis frontalis; 77. Dicranosepsis crinite; 78. Sepsis dissimilis; 79. Sepsis nitens; 80. Coproica ferruginata. 

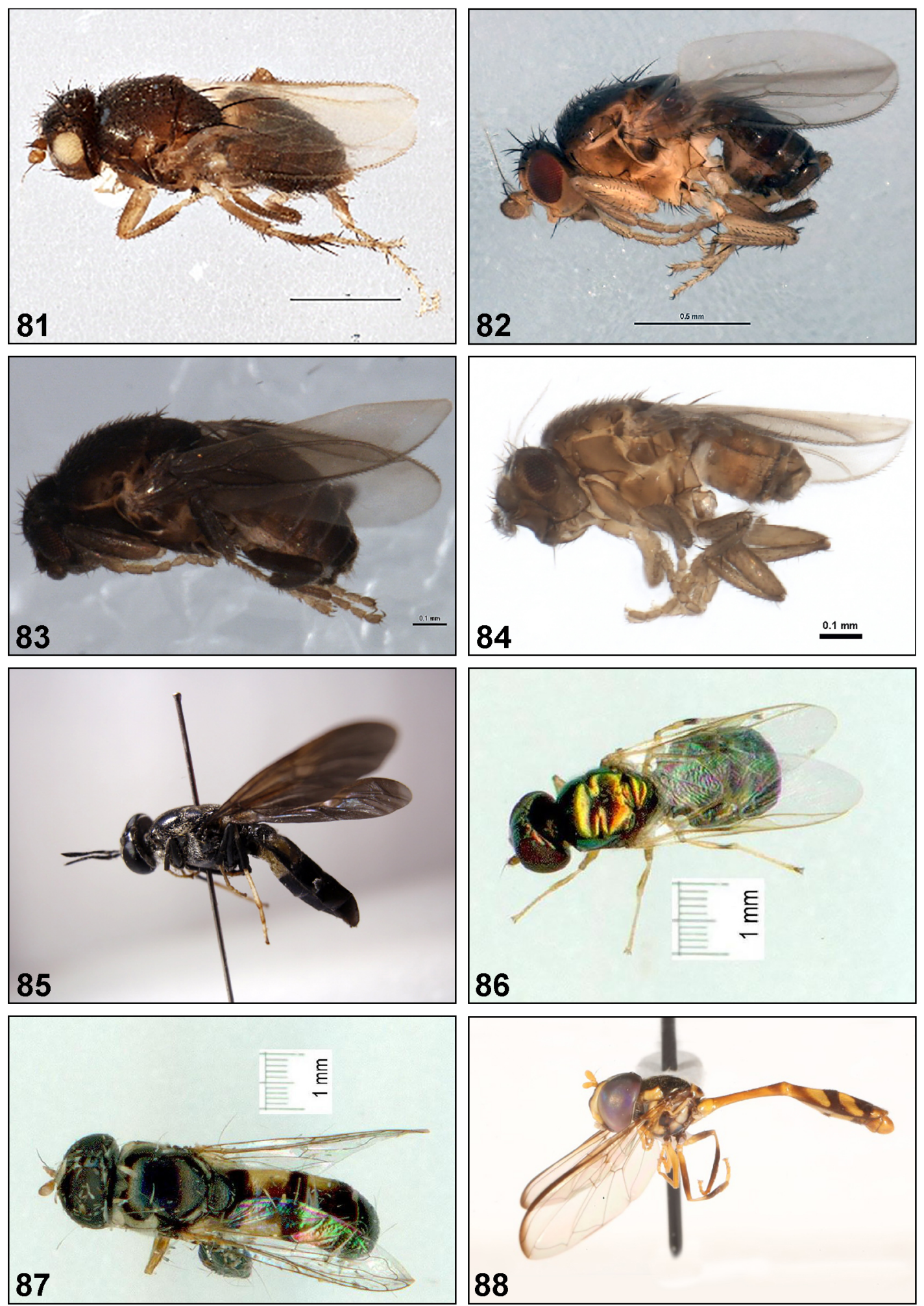

Figures 81-88. General habitus of True flies collected in Bangladesh. 81. Coproica hirtula; 82. Spelobia bifrons; 83. Trachyopella lineafrons; 84. Trachyopella straminea; 85. Hermetia illucens; 86. Microchrysa flaviventris; 87. Syritta orientalis; 88. Allobaccha amphithoe. 

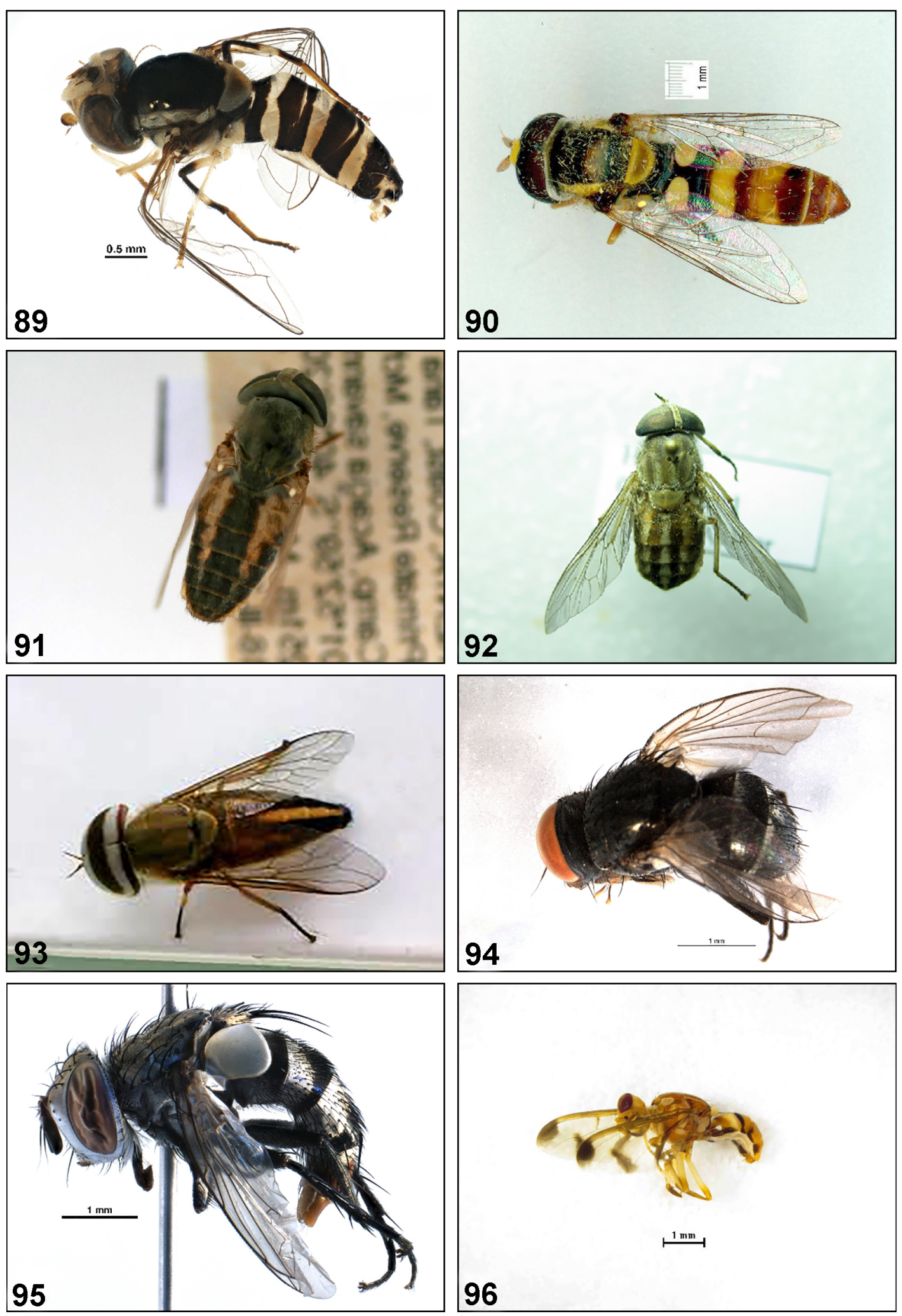

Figures 89-96. General habitus of True flies collected in Bangladesh. 89. Allograpta medanensis; 90. Simosyrphus scutellaris; 91. Atylotus agrestis; 92. Tabanus rubidus; 93. Tabanus striatus; 94. Aplomya metallica; 95. Exorista xanthaspis; 96. Bactrocera cucurbitae. 

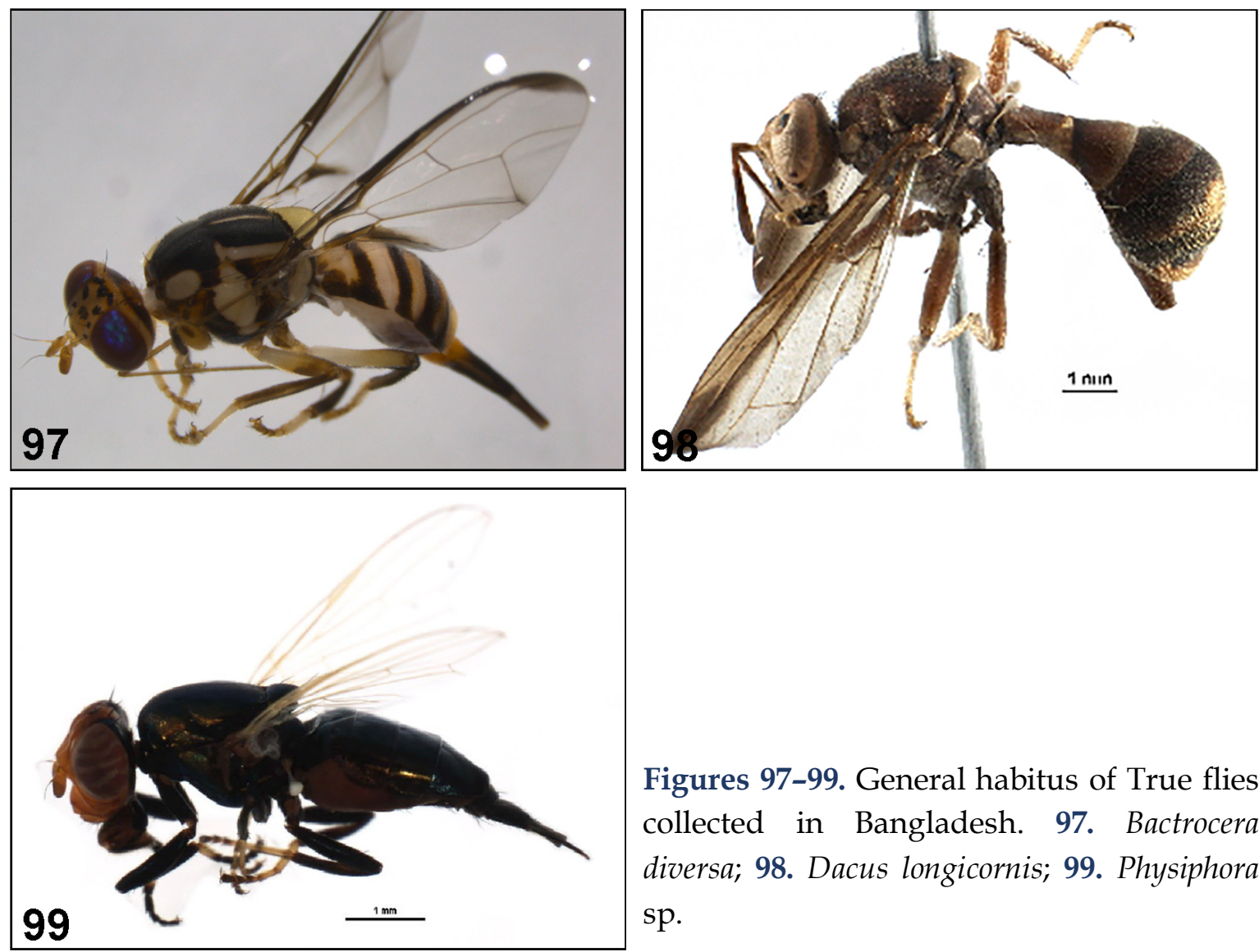

Figures 97-99. General habitus of True flies collected in Bangladesh. 97. Bactrocera diversa; 98. Dacus longicornis; 99. Physiphora sp.

\section{Discussion}

In Bangladesh, some species of three families Micropezidae (Jui et al., 2007; Hill, 2008), Canacidae (Mathis, 1992), and Anthomyiidae (Miah et al., 1997) were reported previously by different authors mentioned in parenthesis but no species were confirmed in the present study. Whilst, various authors were reported varieties of true flies that are also found in the current study. For instance, Huda (1981) noted 30 species of genus Anopheles. Afterwards, Huda \& Majumder (1985) recorded Anopheles vagus, Aedes albopictus, Culex tritaeniorhynchus, Cu. vishnui from CUC. Also, Huda (2000) reported that out of 26 species of genus Aedes only Ae. aegypti and Ae. albopictus are responsible for dengue fever. In the present study $A e$. albopictus was confirmed only.

Rahman et al. (1983) reported Melanagromyza phaseoli (Agromyzidae) as dipteran pest on soybean. Ali et al. (1986) evaluated abundance of Culicoides sp. (Ceratopogonidae) and Chironomus sp. (Chironomidae) at different depth range in a pond. Iwasa et al. (1991) reported a new species Toxopoda mordax (Sepsidae) from the country. Kabir et al. (1991) noted five species of Dacus sp. and their 16 host plants. Hill (2008) noted Mimegralla coerubifrons (Micropezidae) as a minor pest of Ginger (Zingiber officinale). Mazumdar et al. (2010, 2014) recorded Allograpta sp. Anopheles sp. (male), Chrysomya megachephala, Musca domestica, Paragus serratus as flower visiting insects. Biswas \& Das (2011) reported Asphondylia sesami as pest of Sesame. Catling \& Islam (2013) recorded Atherigona oryzae, A. falcata, Anatrichus pygmaeus, Atherigona reversura, Pachylophus rufescens and Hydrellia 
philippina from a deepwater Rice field. Four agromyzid leafminers including Liriomyza chinensis, L. sativae, Melanagromyza obtusa and Ophiomyia phaseoli as well as their 17 plant hosts confirmed by Mazumdar \& Bhuiya (2014). Asaduzzaman et al. (2015) identified Culicoides spp. (Ceratopogonidae) as a vector nematodes caused for Onchocercosis affect mainly cattle, and also buffaloes. Mazumdar \& Bhuiya (2016) recorded Cecidomyiidae (unidentified specimens). Bashar et al. (2016) identified Aedes aegypti, Ae. albopictus, Anopheles vagus, Culex gelidus, Cu. tritaeniorhynchus from Dhaka city. Irish et al. (2016) recorded for 123 species of mosquitoes including Anopheles pseudowillmori, Armigeres malayi and Mimomyia luzonensis. Khan et al. (2017) reported six different bamboo-shoot fly species like Felderimyia gombakensis, Rioxoptilona dunlopi, $R$. vaga, Acroceratitis distincta, A. ceratitina and Gastrozona soror from Dhaka. Sultana et al. (2017) studied the association of five mosquito species namely Aedes aegypti, Ae. albopictus, Armigeres subalbatus and Culex tritaeniorhynchus with their breeding habitat diversity and species distribution in the parks of Dhaka city. Hossain et al. (2017) observed the abundance of Bactrocera zonata in mango orchards. Islam et al. (2017) reported Lucilia cuprina, Chrysomya sp., Musca domestica as Dipteran fly pests of dried fishes at the Sonadia offshore island, Cox's Bazar. Mazumdar (2019) reported Medetera sp. (Medeterinae) from Chittagong City.

By applying DNA barcoding in Bangladesh, Amin et al. (2014) conformed leafminer pest Liriomyza sativae (Agromyzidae). Alam et al. (2015) identified four Drosophila species like Drosophila ananassae, D. parabipectinata, D. repleta and D. melanogaster. Leblanc et al. (2019) surveyed for fruit fly, and a new species, 33 new country records and discovery of the highly invasive Bactrocera carambolae. Based on morphological and molecular identification, Siddiki et al. (2019) reported Aedes aegypti, Ae. albopictus and Culex pipiens from Chittagong city. Mazumdar (2020) ensured Ae. albopictus from Chittagong University Campus through DNA barcoding technique.

The present endeavor was to assess the dipterans diversity by applying DNA barcoding of Malaise trap samples. The results of the current study may a taxonomic baseline study of agro-dipterans for further research.

\section{Acknowledgments}

The authors are extremely grateful to associates at the Centre for Biodiversity Genomics, University of Guelph, for supporting with sequence analysis.

\section{Conflict of Interests}

The authors declare that there is no conflict of interest regarding the publication of this paper.

\section{ORCID}

Paul D.N. Hebert: https:/ / orcid.org/0000-0002-3081-6700

\section{References}

ADCF. (2020) Flee vs. Fly. Available from: https://askdifference.com/flee-vs-fly [Accessed 15th January 2020].

Akhtaruzzaman, M., Alam, M. Z. \& Ali-Sardar, M. M. (1999) Suppressing fruit fly infestation by bagging cucumber at different days after anthesis. Bangladesh Journal of Entomology, 9, 103-112 
Alam, M.S., Ahmed, K.A., Begum, R. \& Shahjahan, R. (2015) Identification of Drosophila species based on 16S rRNA and CO1 gene sequences. Available from: https://researchgate.net/publication/303495952_Identification_of_Drosophila_species_based_ on_16S_rRNA_and_CO1_gene_sequences [Accessed 17th January 2020].

Alam, M.S., Chakma, S., Khan, W.A., Glass, G.E., Mohon, A.N., Elahi, R., Norris, L.C., Podder, M.P., Ahmed, S., Haque, R. \& Sack, D.A. (2012) Diversity of anopheline species and their Plasmodium infection status in rural/ Bandarban, Bangladesh. Parasites \& Vectors, 5 (1), 150. https://doi.org/10.1186/1756-3305-5-150

Alam, M.S., Wagatsuma, Y., Mondal, D., Khanum, H. \& Haque, R. (2009) Relationship between sand fly fauna and kala-azar endemicity in Bangladesh. Acta Tropica, 112 (1), 23-25. https://doi.org/10.1016/j.actatropica.2009.05.021

Alfred, J.R.B. (1999) Fauna of Meghalaya (Part-6). Available from: https://faunaofindia.nic.in/ PDFVolumes/sfs/024/index.pdf [Accessed 22nd February 2020].

Ali, M.M., Islam, M.A. \& Habib, M.A.B. (1986) Monthly and depthwise distribution of benthic fauna in a pond. Bangladesh Journal of Fisheries (Bangladesh), 9 (1-2), 15-21

Ameen, M. \& Moizuddin, M. (1975) Duration of the various developmental stages of Culex pipiens fatigans Wiedemann (Diptera: Culicidae) in Dacca City. Bangladesh Journal of Zoology, 1, 11-22.

Amin, S., Scheffer, S.J., Lewis, M.L., Pasha, M.K. \& Bhuiya, B.A. (2014) DNA barcoding of the vegetable leafminer Liriomyza sativae Blanchard (Diptera: Agromyzidae) in Bangladesh. DNA Barcodes, 2 (1), 29-33

Arabyat, S., Katbeh-Bader, A. \& Greathead, D. (2004) The bee flies (Diptera: Bombyliidae) of Jordan. Zootaxa, 654(1), 1-48. https://doi.org/10.11646/zootaxa.654.1.1

Asaduzzaman, M., Mamun, M.A.A., Anisuzzaman, M., Alim, M.A., Yasin, M.G., Begum, N. \& Mondal, M.M.H. (2015) Epidemiology and pathology of onchocercosis of cattle in Bangladesh. Progressive Agriculture, 26 (2), 147-154. https:// doi.org/10.3329/ pa.v26i2.25976

Bashar, K., Rahman, M.S., Nodi, I.J. \& Howlader, A.J. (2016) Species composition and habitat characterization of mosquito (Diptera: Culicidae) larvae in semi-urban areas of Dhaka, Bangladesh. Pathogens and Global health, 110 (2), 48-61.

https://doi.org/10.1080/20477724.2016.1179862

Biswas, G.C. \& Das, G.P. (2011) Insect and mite pests diversity in the oilseed crops ecosystems in Bangladesh. Bangladesh Journal of Zoology, 39 (2), 235-244. https://doi.org/10.3329/bjz.v39i2.10594

Catling, D. \& Islam, Z. (2013) Diversity and seasonal fluctuations of arthropod fauna in Bangladesh deepwater rice. Bangladesh Rice Journal, 17(1-2), 75-104. https://doi.org/10.3329/brj.v17i1-2.20903

Courtney, G.W., Pape, T., Skevington, J.H. \& Sinclair, B.J. (2017) Biodiversity of Diptera. In: Foottit, R.G. \& Adler, P.H. (eds.) Insect biodiversity: science and society, John Wiley \& Sons: Chichester, UK, 2017; Volume I, pp. 229-278.

deWaard, J.R., V. Levesque-Beaudin, S.L. deWaard, N.V. Ivanova, J.T. McKeown, R. Miskie, S. Naik, K.H. Perez, S. Ratnasingham, C.N. Sobel \& Sones, J.E. (2019) Expedited assessment of terrestrial arthropod diversity by coupling Malaise traps with DNA barcoding. Genome, 62 (3), 85-95. https://doi.org/10.1139/gen-2018-0093

Dhamorikar, A.H. (2017) Flies matter: a study of the diversity of Diptera families (Insecta: Diptera) of Mumbai Metropolitan Region, Maharashtra, India, and notes on their ecological roles. Journal of Threatened Taxa, 9 (11), 10865-10879. https:// doi.org/10.11609/jott.2742.9.11.10865-10879

EBO. (2014) Insect. Available from: https://en.banglapedia.org/index.php?title=Insect [Accessed 18th January 2020]. 
EBO. (2015) Livestock. Available from: https://en.banglapedia.org/index.php?title=Livestock [Accessed 18th January 2020].

Gapud, V.P. (1992) Insect \& mite pests of plant crops in Bangladesh \& their enemies: a compendium. United States Agency for International Development/Bangladesh Agricultural Research Council/CHECCI \& Co. Consulting Inc., pp. 95-99.

Hebert, P.D., Cywinska, A., Ball, S.L. \& Dewaard, J.R. (2003) Biological identifications through DNA barcodes. Proceedings of the Royal Society of London. Series B: Biological Sciences, 270 (1512), 313321. https://doi.org/10.1098/rspb.2002.2218

Hebert, P.D., Ratnasingham, S., Zakharov, E.V., Telfer, A.C., Levesque-Beaudin, V., Milton, M.A., Pedersen, S., Jannetta, P. \& deWaard, J.R. (2016) Counting animal species with DNA barcodes: Canadian insects. Philosophical Transactions of the Royal Society B: Biological Sciences, 371 (1702), 20150333. https://doi.org/10.1098/rstb.2015.0333

Hill, D.S. (2008) Pests of crops in warmer climates and their control. Springer Science \& Business Media. 708 pp.

Hossain, M.A., Momen, M., Uddin, M.S., Khan, S.A. \& Howlader, A.J. (2017) Abundance of peach fruit fly, Bactrocera zonata (Saunders) in mango orchard. Bangladesh Journal of Entomology, 27, 25-34.

Huda, K.M.N. (1981) (April 17). Mosquitoes: Problems and Solutions (in Bangla). The Saptahik Bichitra, Dhacca, Bangladeshpp. 1-6.

Huda, K.M.N. (2000) (August 14) Dengue mosquitoes (in Bangla). The daily Azadi, Chittagong, Bangladesh, pp. 1-4.

Huda, K.M.N. \& Majumder, R. (1985) Observations on Mosquitoes breeding in and around the Chittagong University Campus, Bangladesh. Journal of the Malaysian Society of Health, 5(2), 7578.

Irish, S.R., Al-Amin, H.M., Alam, M.S. \& Harbach, R.E. (2016) A review of the mosquito species (Diptera: Culicidae) of Bangladesh. Parasites \& Vectors, 9 (1), 559. https://doi.org/10.1186/s13071-016-1848-z

Islam (2015) Tachinidae. Available from: https://www.archive.saulibrary.edu.bd:8080/xmlui/bitstream/handle/123456789/1530/Reg. \%2014-06350.pdf?sequence=1\&isAllowed=y [Accessed 19th January 2020].

Islam, A.T.M.F., Islam, M.H., Hossain, T., Yasmin, M., Majumder, M.Z.R., Begum, M. \& Saifullah, A.S.M. (2017) Evaluation of fly population infesting dried fishes at Sonadia Island, Cox's Bazar as a prerequisite for the practical application of sterile insect technique (SIT). Nuclear Science and Applications, 23, 67-70.

Iwasa, M., Zuska, J. \& Ozerov, A.L. (1991) The Sepsidae from Bangladesh, with description of a new species (Diptera). Medical Entomology and Zoology, 42 (3), 229-234.

https://doi.org/10.7601/mez.42.229

Jui, S. M.Z., Alam, M.N., Islam, M.A.K. M. \& Akanda, M. (2007) Field screening of some ginger genotypes for resistance to the rhizome fly, Mimegralla coeruleifrons (Diptera: Micropezidae). Annals of Bangladesh Agriculture, 11(1), 113-124.

Kabir, S.M.H., Rahman, R. \& Molla, M.A.S. (1991) Host plants of dacine fruit flies (Diptera: Tephritidae) of Bangladesh. Bangladesh Journal of Entomology, 1, 69-75.

Karlsson, D., Hartop, E., Forshage, M., Jaschhof, M. \& Ronquist, F. (2020) The Swedish Malaise trap project: a 15 year retrospective on a countrywide insect inventory. Biodiversity Data Journal, 8, e47255. https://doi.org/10.3897/BDJ.8.e47255

Khan, M. (2009) First record of fruit fly, Dacus longicornis Wiedemann (Diptera: Tephritidae) from Bangladesh. Insect Pest Control Newsletter, 72, 33. 
Khan, M., Bari, M.A., Hossain, M., Kovac, D., Freidberg, A., Royer, J. \& Hancock, D.L. (2017) A preliminary survey of bamboo-shoot fruit flies (Diptera: Tephritidae: Acanthonevrini, Gastrozonini), with four new records from Bangladesh. Academic Journal of Entomology, 10(1), 14. https://doi.org/10.5829/idosi.aje.2017.01.04

Leblanc, L., Hossain, M.A., Doorenweerd, C., Khan, S.A., Momen, M., San Jose, M. \& Rubinoff, D. (2019) Six years of fruit fly surveys in Bangladesh: a new species, 33 new country records and discovery of the highly invasive Bactrocera carambolae (Diptera, Tephritidae). ZooKeys, 876, 87109. https://doi.org/10.3897/zookeys.876.38096

Leblanc, L., Hossain, M.A., Khan, S.A., San Jose, M. \& Rubinoff, D. (2013) A preliminary survey of the fruit flies (Diptera: Tephritidae: Dacinae) of Bangladesh. Proceedings of the Hawaiian Entomological Society, 45, 51-58.

Lin, X.L., Stur, E. \& Ekrem, T. (2018) DNA barcodes and morphology reveal unrecognized species in Chironomidae (Diptera). Insect Systematics \& Evolution, 49 (4), 329-398.

https://doi.org/10.1163/1876312X-00002172

Maity, A., Naskar, A., Mukhopadhyay, E., Hazra, S., Sengupta, J., Ghosh, S., \& Banerjee, D. (2015) Taxonomic studies on Tabanidae (Insecta: Diptera) from Himachal Pradesh, India. International Journal of Fauna and Biological Studies, 2 (4), 43-52

Mathis, W.N., (1992) World catalog of the beach-fly family Canacidae (Diptera). Smithsonian Contributions to Zoology, 1-18. https:// doi.org/10.5479/si.00810282.536

Mazumdar, S. (2019) Fauna of Sulakbahar Ward. In: Bhuiya, B.A., Mrida, A.U., Miah, M.I., Hossain, M.K., Siddique, N.A. \& Mannan, S.A. (eds.) Biodiversity: Survey and Conservation of Ward. No. 8, Shulakbahar, 2018, Chattagram City Corporation (CCC), BRGB, pp. 71-164.

Mazumdar, S. (2020) Take precautions against Aedes mosquitoes. Available from: https://smazumdar.com/2020/04/take-precautions-against-aedes.html [Accessed 3rd September 2020].

Mazumdar, S, Bhuiya, B.A. \& Pasha, M.K. (2010) A checklist of insect foraging and pollinators of crops of Bangladesh. Journal of Taxonomy and Biodiversity Research, 4 (1), 35-40

Mazumdar, S, Bhuiya, B.A. \& Pasha, M.K. (2011) Pollinating True flies (Insecta: Diptera) and their foraging behavior of seasonal crops of Bangladesh. Journal of Taxonomy and Biodiversity Research, $5,7-10$

Mazumdar, S. \& Bhuiya, B.A., (2014) Vegetable leafminers (Diptera: Agromyzidae) and their plant hosts in Bangladesh. Journal of Threatened Taxa, 6 (6), 5894-5899. https://doi.org/10.11609/JoTT.03892.5894-9

Mazumdar, S. \& Bhuiya, B.A., (2016) Parasitoids (Hymenoptera) of leafminer flies (Diptera: Agromyzidae) from Bangladesh. Journal of Threatened Taxa, 8 (4), 8714-8718.

https://doi.org/10.11609/jott.2741.8.4.8714-8718

Mazumdar, S., Bhuiya, B.A. \& Pasha, M.K. (2014) Diversity of flower visiting insects of crops in Bangladesh. LEPCEY - The Journal of Tropical Asian Entomology, 3 (1), 33-47

Mazumdar, S., Hebert, P.D.N., Bhuiya, B.A. \& Miah, M.I. (2019) Parasitic Hymenoptera Recovered by DNA Barcoding of Malaise Trap Collection at the Chittagong University Campus, Bangladesh. American Journal of BioScience, 7 (6), 94-98.

https://doi.org/10.11648/j.ajbio.20190706.12

Miah, M.R.U., Sarkar, R., Alam, S.M.K. \& Islam, B.N. (1997) Screening of prosomillet genotypes against shootfly (Atherigona sp; Anthomyiidae: Diptera). Bangladesh Journal of Zoology, 25, 91-94.

Pape, T. (1996) Catalogue of the Sarcophagidae of the world (Insecta: Diptera). Associated Publishers. 558 pp.

Rahman, M., Mannan, M.A. \& Jahan, S., (1983) Insect problems on soybean in Bangladesh. In 8. Bangladesh Science Conference, Dhaka (Bangladesh), 5-9 Feb 1983. BAAS. 
Rahman, M.M., Howlader, M.T.H., Islam, K.S. \& Morshed, M.N. (2019) Efficacy of three biopesticides against cucurbit fruit fly, Bactrocera cucurbitae Coquillett (Diptera: Tephritidae) and yield of bitter gourd. Journal of the Bangladesh Agricultural University, 17 (4), 483-489. https://doi.org/10.3329/jbau.v17i4.44616

Rain, F.F., Howlader,A., \& Aslam, A.F.M. (2019) Molecular identification and characterization of medically and veterinary important flies of Bangladesh based on mitochondrial COI gene sequences. Asia Pacific Journal of Molecular Biology and Biotechnology, 69-79.

https://doi.org/10.35118/apjmbb.2019.027.4.08

Sharma, M., Singh, P. \& Singh, D. (2017) Morphological studies on Parasarcophaga albiceps, Parasarcophaga macroauriculata and Parasarcophaga ruficornis (Diptera: Sarcophagidae) of Indian origin. International Journal of Fauna and Biological Studies, 4(4), 155-161.

Siddiki, A.M.A.M.Z.,Sarker, M.S., Mazumder, S., Bhuiya, B.A., Basher, K., Kamal, T. \& Hossain, M.A. (2019) Morphotaxonomic and DNA barcoding analyses of mosquitoes collected from Chattogram Metropolitan area. Bangladesh Journal of Veterinary and Animal Sciences, 7(1), 1-08.

Sitompul, A.F., Siregar, E.H., Roesma, D.I., Dahelmi, D. \& Prasetya, E. (2018) Molecular identification of coffee (Coffea arabica) pollinator insects in North Sumatra, Indonesia based on designed COI primers. Biodiversitas Journal of Biological Diversity, 19 (5), 1876-1883. https://doi.org/10.13057/biodiv/d190539

SMT. (2020) Flesh Flies (Diptera: Sarcophagidae). Available from: https://sarcophagidae.myspecies.info/ taxonomy/term/2519/descriptions [Accessed 8th March 2020].

Sultana, A., Hasan, S., Hossain, M., Alim, A., Al Mamun, M. \& Bashar, K. (2017) Larval breeding habitats and ecological factors influence the species composition of mosquito (Diptera: Culicidae) in the parks of Dhaka city, Bangladesh. Bangladesh Journal of Zoology, 45(2), 111-122. https://doi.org/10.3329/bjz.v45i2.35706 
بررسى دوبالان (Insecta: Diptera) جمع آورى شده توسط تله مالايز با استفاده از DNA باركدينَ در بنَلادش

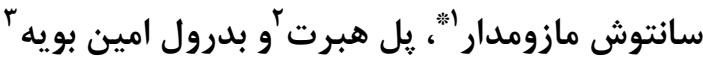

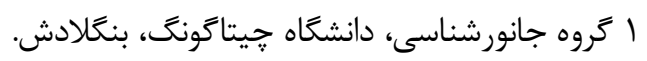

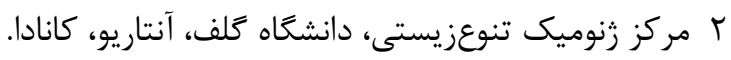

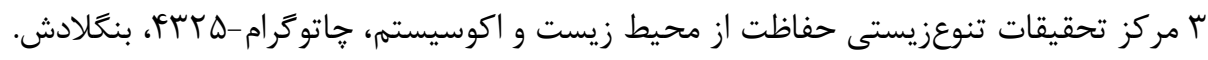

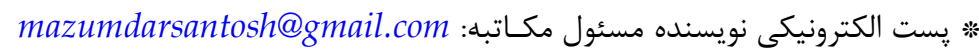

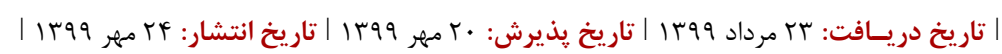

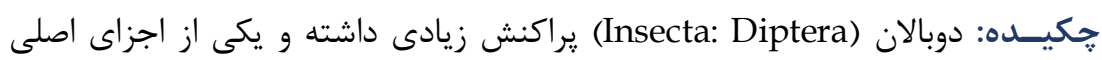
اغلب اكوسيستمها هستند. هدف از اين مطالعه، شناسايى تنوع دوبالان از طريق إنانيق

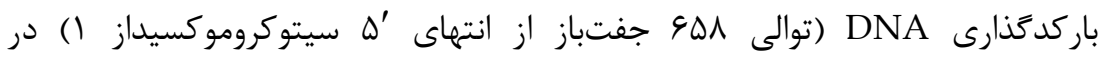

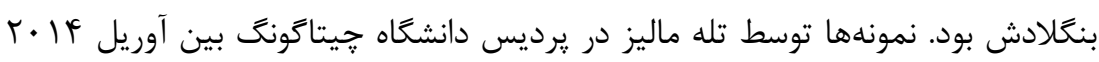

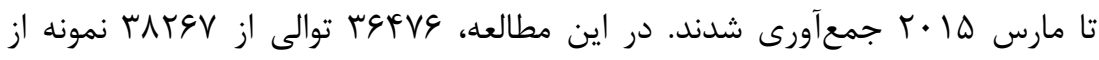

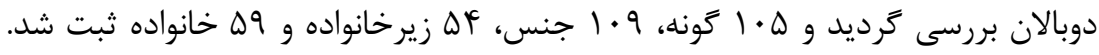

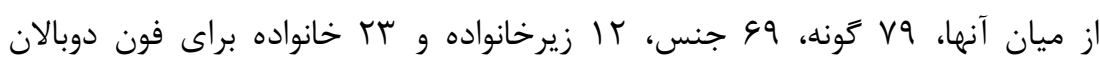

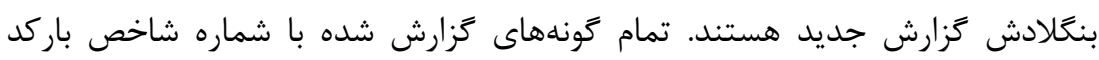
(BOLD) Barcode of Life Data System (يروكسى هاى تونه) در سامانه (BINs) موجود است. وازَتــان كليدى: دوبالان، تله ماليز، باركد DNA، بنَكَلادش 